\title{
ADJOINT FUNCTORS
}

BY

\author{
DANIEL M. KAN(1)
}

1. Introduction. In homology theory an important role is played by pairs of functors consisting of

(i) a functor Hom in two variables, contravariant in the first variable and covariant in the second (for instance the functor which assigns to every two abelian groups $A$ and $B$ the group $\operatorname{Hom}(A, B)$ of homomorphisms $f: A \rightarrow B)$.

(ii) a functor $\otimes$ (tensor product) in two variables, covariant in both (for instance the functor which assigns to every two abelian groups $A$ and $B$ their tensor product $A \otimes B$ ).

These functors are not independent; there exists a natural equivalence of the form

$$
\alpha: \operatorname{Hom}(\otimes,) \rightarrow \operatorname{Hom}(, \operatorname{Hom}(,))
$$

Such pairs of functors will be the subject of this paper.

In the above formulation three functors Hom and only one tensor product are used. It appears however that there exists a kind of duality between the tensor product and the last functor Hom, while both functors Hom outside the parentheses play a secondary role.

Let $\mathfrak{M}$ be the category of sets. For each category $y$ let $H: \mathcal{Y}, \mathcal{Y} \rightarrow \mathfrak{M}$ be the functor which assigns to every two objects $A$ and $B$ in $y$ the set $H(A, B)$ of the maps $f: A \rightarrow B$ in $Y$.

Let $X$ and $\mathrm{Z}$ be categories and let $S: X \rightarrow \mathcal{Z}$ and $T: \mathcal{Z} \rightarrow X$ be covariant functors. Then $S$ is called a left adjoint of $T$ and $T$ a right adjoint of $S$ if there exists a natural equivalence

$$
\alpha: H(S(x), \mathrm{Z}) \rightarrow H(x, T(\mathrm{Z})) .
$$

An important property of adjoint functors is that each determines the other up to a unique natural equivalence.

Examples of adjoint functors are:

(i) Let $a$ be the category of topological spaces; then the functor $\times I: Q \rightarrow Q$ which assigns to every space its cartesian product with the unit interval $I$ is a left adjoint of the functor $\Omega: Q \rightarrow Q$ which assigns to every space the space of all its paths.

(ii) Let $S$ be the category of c.s.s. complexes; then the simplicial singular functor $S: Q \rightarrow S$ is a right adjoint of the realization functor $R: S \rightarrow Q$ which assigns to every c.s.s. complex $K$ a CW-complex of which the $n$-cells are in one-to-one correspondence with the nondegenerate $n$-simplices of $K$.

Received by the editors September 20, 1956.

(1) The author is now at The Hebrew University in Jerusalem. 
The notion of adjointness may be generalized to functors in two (or more) variables; a covariant functor $S: \mathbb{X}, \mathcal{Y} \rightarrow \mathrm{Z}$ is called a left adjoint of a functor $T: \mathcal{Y}, \mathcal{Z} \rightarrow X$, contravariant in $\mathcal{Y}$ and covariant in $\mathcal{Z}$, and $T$ is called a right adjoint of $S$ if there exists a natural equivalence

$$
\alpha: H(S(x, y), \mathrm{Z}) \rightarrow H(x, T(\mathcal{Y}, \mathrm{Z})) .
$$

Adjoint functors in two (or more) variables also determine each other up to a unique natural equivalence. The situation is similar when both functors $H$ are replaced by other functors.

An example of adjoint functors in two variables are the functors $\otimes$ and Hom mentioned above; $\otimes$ is a left adjoint of Hom.

The general theory of adjoint functors constitutes Chapter I.

In Chapter II we deal with direct and inverse limits. It is shown that a direct limit functor (if such exists) is a left adjoint of a certain functor which always can be defined, while an inverse limit functor is a right adjoint of a similar functor.

In Chapter III several existence theorems are given. In [2] a procedure was described by which from a given functor new functors, called lifted, can be derived. Let the functor $S: \mathbb{X}, \mathcal{Y} \rightarrow \mathrm{Z}$ be a left adjoint of the functor $T: \mathcal{Y}, \mathrm{Z} \rightarrow \mathfrak{X}$, then sufficient conditions will be given in order that a lifted functor of $S$ has a right adjoint or that a lifted functor of $T$ has a left adjoint. Thus sometimes starting from a given pair of adjoint functors, new such pairs may be constructed; for instance starting from the adjoint functors $\otimes$ and Hom on abelian groups, pairs of adjoint functors involving groups with operators, chain complexes, etc. may be obtained.

A category $\mathrm{Z}$ is always accompanied by the functor $H: \mathrm{Z}, \mathrm{Z} \rightarrow \mathfrak{M}$ and its lifted functors. A necessary and sufficient condition in order that all these functors have a left adjoint is that a notion of direct limit can be defined in $\mathrm{Z}$. Several known functors involving c.s.s. complexes can be obtained either by lifting of a suitable functor $H$ or from a left adjoint of such a lifted functor. These applications will be dealt with a sequel entitled Functors involving c.s.s. complexes [5].

I am deeply grateful to S. Eilenberg for his helpful criticism during the preparation of this paper.

\section{Chapter I. General theory}

2. Notation and terminology. For the definition of the notions category, functor, natural transformation, etc. see [2].

A functor $F$ defined on the categories $y_{1}, \cdots, y_{n}$ will often be denoted by $F\left(\mathcal{Y}_{1}, \cdots, \mathcal{Y}_{n}\right)$. Similarly if $F$ and $G$ are functors defined on the categories $\mathcal{Y}_{1}, \cdots, Y_{n}$, then a natural transformation $\alpha: F \rightarrow G$ is sometimes denoted by $\alpha\left(Y_{1}, \cdots, y_{n}\right)$.

Only categories will be considered which satisfy the following condition

Condition 2.1. For every two objects $A$ and $B$ in a category $y$ the maps 
$f: A \rightarrow B$ in $\mathcal{Y}$ form a set, denoted by $H(A, B)$.

Clearly the category $\mathfrak{M}$ of sets satisfies condition 2.1 .

Let $a: A^{\prime} \rightarrow A$ and $b: B \rightarrow B^{\prime}$ be maps in a category $y$ and let

$$
H(a, b): H(A, B) \rightarrow H\left(A^{\prime}, B^{\prime}\right)
$$

denote the set mapping defined by

$$
H(a, b) f=b \circ f \circ a \quad f \in H(A, B) .
$$

Then it is easily verified that

Proposition 2.2. The function $H: \mathcal{Y}, \mathcal{Y} \rightarrow \mathfrak{M}$ is a functor, contravariant in the first variable and covariant in the second.

Hence every category $y$ is accompanied by a functor

$$
H: \mathcal{Y}, \mathcal{Y} \rightarrow \mathfrak{T} \text {. }
$$

A category $v$ will be called proper if its objects form a set. Clearly in view of condition 2.1 the maps of a proper category form also a set.

The dual of a category $y$ is the category $y^{*}$ which has the same objects and maps as $\mathcal{Y}$; however

(a) an object $A \in \mathcal{Y}$ is denoted by $A^{*}$ if it is considered as an object of $\mathrm{Y}^{*}$,

(b) a map $f: A \rightarrow B \in \mathcal{Y}$ is denoted by $f^{*}: B^{*} \rightarrow A^{*}$ if it is considered as a map of $\mathcal{Y}^{*}$, and

(c) the composition of two maps $f^{*}: B^{*} \rightarrow A^{*}$ and $g^{*}: C^{*} \rightarrow B^{*}$ in $y^{*}$ is defined by $f^{*} \circ g^{*}=(g \circ f)^{*}$.

Clearly $y^{* *}=y$ and $H(A, B)=H\left(B^{*}, A^{*}\right)$ for every two objects $A$ and $B$ in $y$.

The dual of a functor $F\left(Y_{1}, \cdots, Y_{n}\right)$ is the functor $F^{*}\left(Y_{1}^{*}, \cdots, Y_{n}^{*}\right)$ defined by

$$
\begin{aligned}
F^{*}\left(Y_{1}^{*}, \cdots, Y_{n}^{*}\right) & =F\left(Y_{1}, \cdots, Y_{n}\right)^{*}, \\
F^{*}\left(y_{1}^{*}, \cdots, y_{n}^{*}\right) & =F\left(y_{1}, \cdots, y_{n}\right)^{*}
\end{aligned}
$$

for every object $Y_{i} \in \mathcal{Y}_{i}$ and every map $y_{i} \in \mathcal{Y}_{i}$.

3. Adjoint functors in one variable.

Definition (3.1). Let $X$ and $\mathrm{Z}$ be categories, let $S: \mathbb{X} \rightarrow \mathrm{Z}$ and $T: \mathrm{Z} \rightarrow \mathbb{X}$ be covariant functors and let

$$
\alpha: H(S(x), \mathrm{Z}) \rightarrow H(x, T(\mathrm{z}))
$$

be a natural equivalence. Then $S$ is called the left adjoint of $T$ under $\alpha$ and $T$ the right adjoint of $S$ under $\alpha$ (Notation $\alpha: S \dashv T$ ).

An important property of two adjoint functors is that each of them determines the other up to a unique natural equivalence. This is expressed by the following uniqueness theorems. 
TheOREM 3.2. Let $S, S^{\prime}: \mathrm{X} \rightarrow \mathrm{Z}$ and $T, T^{\prime}: \mathrm{Z} \rightarrow \mathrm{X}$ be covariant functors and let $\alpha: S \dashv T$ and $\alpha^{\prime}: S^{\prime} \dashv T^{\prime}$. Let $\sigma: S^{\prime} \rightarrow S$ be a natural transformation. Then there exists a unique natural transformation $\tau: T \rightarrow T^{\prime}$ such that commutatively holds in the diagram

$$
\begin{aligned}
& H(S(\mathfrak{X}), \mathrm{Z}) \stackrel{\alpha}{\longrightarrow} H(x, T(\mathrm{Z})) \\
& \begin{aligned}
& \downarrow H(\sigma(x), \mathrm{Z}) \downarrow H(X, \tau(\mathrm{Z})) \\
& H\left(S^{\prime}(\mathfrak{X}), \mathrm{Z}\right) \stackrel{\alpha^{\prime}}{\longrightarrow} H\left(X, T^{\prime}(\mathrm{Z})\right)
\end{aligned}
\end{aligned}
$$

If $\sigma$ is a natural equivalence, then so is $\tau$.

THEOREM 3.2*. Let $S, S^{\prime}: \mathrm{X} \rightarrow \mathrm{Z}$ and $T, T^{\prime}: \mathrm{Z} \rightarrow \mathfrak{X}$ be covariant functors and let $\alpha: S \dashv T$ and $\alpha^{\prime}: S^{\prime} \dashv T^{\prime}$. Let $\tau: T \rightarrow T^{\prime}$ be a natural transformation. Then there exists a unique natural transformation $\sigma: S^{\prime} \rightarrow S$ such that commutativity holds in the diagram (3.2a). If $\tau$ is a natural equivalence, then so is $\sigma$.

Proof of Theorem 3.2. Suppose $\tau: T \rightarrow T^{\prime}$ is a natural transformation such that commutativity holds in 3.2a. Then for every object $X \in X$ and $Z \in Z$ and for every map $f \in H(X, T Z)$

$$
\tau Z \circ f=H(X, \tau Z) f=\alpha^{\prime}\left(H(\sigma X, Z) \alpha^{-1} f\right)=\alpha^{\prime}\left(\alpha^{-1} f \circ \sigma X\right) .
$$

In particular if $X=T Z$ and $f=i_{T Z}$, then

$$
\tau Z=\alpha^{\prime}\left(\alpha^{-1} i_{T Z} \circ \sigma T Z\right) .
$$

Consequently if a natural transformation $\tau: T \rightarrow T^{\prime}$ exists such that commutativity holds in $3.2 \mathrm{a}$, then it must satisfy (3.3). Hence it is unique.

It follows from the naturality of $\alpha^{\prime}$ that for every map $g: Z \rightarrow Z^{\prime} \in \mathcal{Z}$ commutativity holds in the diagrams
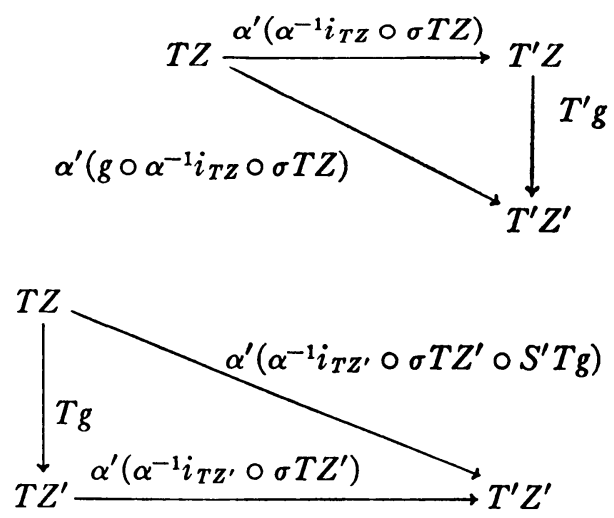

and in view of the naturality of $\alpha$ and $\sigma$ commutativity also holds in the diagram 


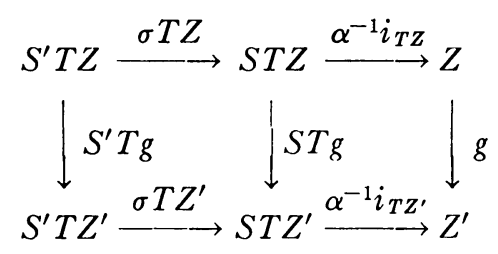

Consequently

$$
\begin{aligned}
\tau Z^{\prime} \circ T g & =\alpha^{\prime}\left(\alpha^{-1} i_{T Z^{\prime}} \circ \sigma T Z^{\prime}\right) \circ T g \\
& =\alpha^{\prime}\left(\alpha^{-1} i_{T Z^{\prime}} \circ \sigma T Z^{\prime} \circ S^{\prime} T g\right) \\
& =\alpha^{\prime}\left(g \circ \alpha^{-1} i_{T Z} \circ \sigma T Z\right) \\
& =T^{\prime} g \circ \alpha^{\prime}\left(\alpha^{-1} i_{T Z} \circ \sigma T Z\right)=T^{\prime} g \circ \tau Z^{\prime},
\end{aligned}
$$

i.e. the function $\tau$ defined by (3.3) is a natural transformation.

Now let $\sigma$ be a natural equivalence and let $\tau^{\prime}$ be the natural transformation induced by $\sigma^{-1}$. Then $\tau \tau^{\prime}$ and $\tau^{\prime} \tau$ are natural transformations induced by $\sigma \sigma^{-1}$ and $\sigma^{-1} \sigma$, and the uniqueness of $\tau \tau^{\prime}$ and $\tau^{\prime} \tau$ together with the fact that $\sigma \sigma^{-1}$ and $\sigma^{-1} \sigma$ are identities yields that $\tau \tau^{\prime}$ and $\tau^{\prime} \tau$ are also identities, i.e. $\tau$ is a natural equivalence with inverse $\tau^{\prime}$. This completes the proof of Theorem 3.2 .

The proof of Theorem $3.2^{*}$ is similar. Theorem $3.2^{*}$ could also have been obtained from Theorem 3.2 using the duality Theorem 3.4 below, which essentially asserts that a functor $S$ is a left adjoint of a functor $T$ if and only if the functor $S^{*}$, the dual of $S$, is a right adjoint of the functor $T^{*}$, the dual of $T$.

Let $S: X \rightarrow Z$ and $T: Z \rightarrow X$ be covariant functors and let $S^{*}: \mathscr{X}^{*} \rightarrow \mathrm{Z}^{*}$ and $T^{*}: \mathrm{Z}^{*} \rightarrow X^{*}$ be their duals. Then by definition $I I(S X, Z)=H\left(Z^{*}, S^{*} X^{*}\right)$ and $H(X, T Z)=H\left(T^{*} Z^{*}, X^{*}\right)$ for every object $X \in X$ and $Z \in Z$.

Theorem 3.4. Let $\alpha: S(X) \dashv T(\mathcal{Z})$ and define for every object $X^{*} \in X^{*}$ and $Z^{*} \in \mathrm{Z}^{*} a \operatorname{map}$

$$
\alpha^{\sharp}\left(Z^{*}, X^{*}\right): H\left(T^{*} Z^{*}, X^{*}\right) \rightarrow H\left(Z^{*}, S^{*} X^{*}\right)
$$

by

$$
\alpha^{\sharp}\left(Z^{*}, X^{*}\right)=\alpha^{-1}(X, Z) .
$$

Then the function

$$
\alpha^{\#}: H\left(T^{*}\left(\mathrm{Z}^{*}\right), \mathfrak{X}^{*}\right) \rightarrow H\left(\mathrm{Z}^{*}, S^{*}\left(\mathfrak{X}^{*}\right)\right)
$$

is a natural equivalence, i.e. $\alpha^{\#}: T^{*} \dashv S^{*}$. Also $\alpha^{\# \#}=\alpha$.

Proof. I.et $x^{*}: X^{*} \rightarrow X^{*} \in \mathfrak{X}^{*}$ and $z^{*}: Z^{*} \rightarrow Z^{*} \in \mathrm{Z}^{*}$ be maps. Then it follows from the naturality of $\alpha$ that for every map $f^{*} \in I I\left(T^{*} Z^{*}, X^{*}\right)$ 


$$
\begin{aligned}
\alpha^{*}\left(Z^{\prime *}, X^{*}\right) H( & \left.T^{*} z^{*}, x^{*}\right) f^{*}=\alpha^{\sharp}\left(Z^{\prime *}, X^{*}\right)\left(x^{*} \circ f^{*} \circ T^{*} z^{*}\right) \\
= & \left(\alpha^{-1}\left(X^{\prime}, Z^{\prime}\right)(T z \circ f \circ x)\right)^{*}=\left(\alpha^{-1}\left(X^{\prime}, Z^{\prime}\right) H(x, T z) f\right)^{*} \\
= & \left(H(S x, z) \alpha^{-1}(X, Z) f\right)^{*}=\left(z \circ \alpha^{-1}(X, Z) f \circ S x\right)^{*} \\
= & S^{*} x^{*} \circ \alpha^{*}\left(Z^{*}, X^{*}\right) f^{*} \circ z^{*}=H\left(z^{*}, S^{*} x^{*}\right) \alpha^{\sharp}\left(Z^{*}, X^{*}\right) f^{*}
\end{aligned}
$$

i.e. $\alpha^{*}$ is a natural transformation. The fact that $\alpha$ and hence $\alpha^{-1}$ is a natural equivalence now implies that $\alpha^{\#}$ is so. That $\alpha^{\# \#}=\alpha$ follows immediately from 3.4a.

4. Adjoint functors in several variables. A covariant functor $S: \mathfrak{X}, \mathfrak{Y} \rightarrow \mathbb{Z}$ may be regarded as a collection consisting of

(i) a covariant functor $S(, Y): \mathfrak{X} \rightarrow \mathrm{Z}$ for every object $Y \in \mathcal{Y}$ and

(ii) a natural transformation $S(, y): S(, Y) \rightarrow S\left(, Y^{\prime}\right)$ for every map $y: Y \rightarrow$ $Y^{\prime} \in \mathcal{Y}$.

Now suppose that for every object $Y \in \mathcal{Y}$ a covariant functor $T_{Y}: \mathcal{Z} \rightarrow \mathscr{X}$ and a natural equivalence

$$
\alpha_{Y}: H(S(x, Y), \mathrm{Z}) \rightarrow H\left(x, T_{Y}(\mathrm{Z})\right)
$$

are given, i.e. $\alpha_{Y}: S(, Y) \dashv T_{Y}$. Then it follows from Theorem 3.2 that for every map $y: Y \rightarrow Y^{\prime} \in \mathcal{Y}$ there exists a unique natural transformation $T_{y}: T_{Y^{\prime}} \rightarrow T_{Y}$ such that commutativity holds in the diagram

$$
\begin{gathered}
H(S(x, Y), \mathrm{Z}) \stackrel{\alpha_{Y}}{\longrightarrow} H\left(x, T_{Y}(\mathrm{Z})\right) \\
\uparrow H(S(x, y), \mathrm{Z}) \\
H\left(S\left(x, Y^{\prime}\right), \mathrm{Z}\right) \stackrel{\alpha_{Y^{\prime}}}{\longrightarrow} H\left(x, T_{y}(\mathrm{Z})\right)
\end{gathered}
$$

Let $y^{\prime}: Y^{\prime} \rightarrow Y^{\prime \prime} \in \mathcal{Y}$. Then the uniqueness of the natural transformations $T_{y}, T_{y^{\prime}}$ and $T_{y^{\prime} y}$ implies that $T_{y} T_{y^{\prime}}=T_{y^{\prime} y}$. Similarly if $i: Y \rightarrow Y$ is the identity, then $T_{i}: T_{Y} \rightarrow T_{Y}$ is the identity natural transformation. Consequently the function $T$ defined by

$$
\begin{aligned}
T(Y, Z) & =T_{Y} Z, \\
T(y, z) & =T_{Y} z \circ T_{y} Z
\end{aligned}
$$

for every object $Y \in \mathcal{Y}$ and $Z \in Z$ and every map $y: Y \rightarrow Y^{\prime} \in \mathcal{Y}$ and $z: Z \rightarrow Z^{\prime}$ $\in \mathrm{Z}$, is a functor $T: \mathcal{Y}, \mathrm{Z} \rightarrow \mathcal{X}$, contravariant in $\mathcal{Y}$ and covariant in $\mathrm{Z}$.

Clearly the function $\alpha$ defined by

$$
\alpha(X, Y, Z)=\alpha_{Y}(X, Z) .
$$

for every object $X \in X, Y \in \mathcal{Y}$ and $Z \in Z$, is a natural equivalence

$$
\alpha: H(S(x, y), \mathrm{Z}) \rightarrow H(x, T(Y, Z))
$$

Thus we have 
THEOREM 4.1. Let $S: x, \mathcal{Y} \rightarrow \mathbb{Z}$ be a covariant functor and let for every object $Y \in \mathcal{Y}$ be given a covariant functor $T_{Y}: \mathrm{Z} \rightarrow \mathrm{X}$ and a natural equivalence

$$
\alpha_{Y}: H(S(X, Y), \mathrm{Z}) \rightarrow H\left(X, T_{Y}(\mathrm{Z})\right),
$$

i.e. $\alpha_{Y}: S(, Y) \dashv T_{Y}$. Then there exists a unique functor

$$
T: \mathcal{Y}, \mathrm{Z} \rightarrow \mathfrak{X}
$$

contravariant in $y$ and covariant in $\mathrm{Z}$ and a unique natural equivalence

$$
\alpha: H(S(x, y), \mathrm{Z}) \rightarrow H(x, T(Y, z))
$$

such that for every object $X \in X, Y \in \mathcal{Y}$ and $Z \in \mathcal{Z}$

$$
T(Y, Z)=T_{Y} Z, \quad \alpha(X, Y, Z)=\alpha_{Y}(X, Z),
$$

i.e. $\alpha: S \dashv T$.

REMARK 4.2. It is clear that in the above starting from a functor $S: x$, $\mathcal{Y} \rightarrow \mathcal{Z}$, contravariant in $\mathcal{Y}$, a functor $T: \mathcal{Y}, \mathcal{Z} \rightarrow \mathfrak{X}$, covariant in $\mathcal{Y}$, would have been obtained. As however a functor contravariant in $\mathcal{Y}$ becomes covariant when regarded as a functor in $\mathcal{Y}^{*}$, the dual of $\mathcal{Y}$, we may restrict ourselves to functors $S: x, Y \rightarrow Z$ which are covariant in both variables.

In view of Theoem 4.1 and Remark 4.2 we now define adjoint functions in two variables as follows.

Definition 4.3. Let $S: \mathbb{X}, \mathcal{Y} \rightarrow \mathrm{Z}$ be a covariant functor, let $T: \mathcal{Y}, \mathrm{z} \rightarrow \mathfrak{X}$ be a functor contravariant in $\mathcal{Y}$ and covariant in $\mathrm{Z}$ and let

$$
\alpha: H(S(x, Y), Z) \rightarrow H(x, T(Y, Z))
$$

be a natural equivalence. Then $S$ is called the left adjoint of $T$ under $\alpha$ and $T$ the right adjoint of $S$ under $\alpha$ (Notation $\alpha: S \dashv T$ ).

As in the case of functors in one variable, adjoint functors in two variables determine each other up to a unique natural equivalence. This is expressed by the following uniqueness theorems which by the above argument follow directly from the Theorems 3.2 and $3.2 *$.

Theorem 4.4. Let $S, S^{\prime}: \mathfrak{X}, \mathfrak{Y} \rightarrow \mathrm{Z}$ be covariant functors, let $T, T^{\prime}: \mathfrak{Y}, \mathrm{Z} \rightarrow \mathfrak{X}$ be functors contravariant in $Y$ and covariant in $\mathrm{Z}$ and let $\alpha: S \dashv T$ and $\alpha^{\prime}: S^{\prime} \dashv T^{\prime}$. Let $\sigma: S^{\prime} \rightarrow S$ be a natural transformation. Then there exists a unique natural transformation $\tau: T \rightarrow T^{\prime}$ such that commutativity holds in the diagram

$$
\begin{aligned}
& H(S(x, Y), \mathcal{Z}) \stackrel{\alpha}{\longrightarrow} H(x, T(Y, Z)) \\
& \downarrow H(\sigma(x, y), \mathrm{Z}) \quad\lfloor H(x, \tau(\mathcal{Y}, \mathrm{Z})) \\
& H\left(S^{\prime}(\mathfrak{X}, \mathcal{Y}), \mathrm{Z}\right) \stackrel{\alpha}{\longrightarrow} H\left(X, T^{\prime}(\mathcal{Y}, \mathrm{Z})\right)
\end{aligned}
$$

If $\sigma$ is a natural equivalence, then so is $\tau$. 
Theorem $4.4^{*}$. Let $S, S^{\prime}: x, Y \rightarrow Z$ be covariant functors, let $T, T^{\prime}: \mathcal{Y}, \mathrm{Z} \rightarrow \mathfrak{X}$ be functors contravariant in $Y$ and covariant in $\mathrm{Z}$ and let $\alpha: S \dashv T$ and $\alpha^{\prime}: S^{\prime} \dashv T^{\prime}$. Let $\tau: T \rightarrow T^{\prime}$ be a natural transformation. Then there exists a unique natural transformation $\sigma: S^{\prime} \rightarrow S$ such that commutativity holds in the diagram (4.4a). If $\tau$ is a natural equivalence, then so is $\sigma$.

The duality Theorem 3.4 may be generalized as follows. Let $S: x, y \rightarrow Z$ be a covariant functor and denote by $S^{\sharp}: \mathcal{Y}, x^{*} \rightarrow Z^{*}$ the functor contravariant in $\mathcal{Y}$ and covariant in $x^{*}$ defined by

$$
\begin{aligned}
S^{*}\left(Y, X^{*}\right) & =S(X, Y)^{*}, \\
S^{*}\left(y, x^{*}\right) & =S(x, y)^{*}
\end{aligned}
$$

for every object $Y \in \mathcal{Y}$ and $X^{*} \in x^{*}$ and every map $y \in \mathcal{Y}$ and $x^{*} \in x^{*}$. Similarly let $T: \mathcal{Y}, \mathcal{Z} \rightarrow \mathfrak{X}$ be a functor contravariant in $\mathcal{Y}$ and covariant in

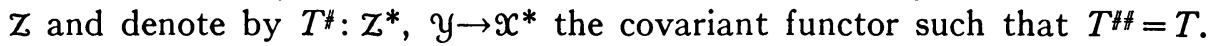
Then clearly for every object $X \in X, Y \in \mathcal{Y}$ and $Z \in \mathcal{Z}$

$$
\begin{aligned}
& H(S(X, Y), Z)=H\left(Z^{*}, S^{\sharp}\left(Y, X^{*}\right)\right) ; \\
& H(X, T(Y, Z))=H\left(T^{\sharp}\left(Z^{*}, Y\right), X^{*}\right) .
\end{aligned}
$$

THEOREM 4.5. Let $\alpha: S(x, Y) \dashv T(Y, Z)$ and define for every object $X^{*} \in \mathfrak{X}^{*}$, $Y \in \mathcal{Y}$ and $Z^{*} \in Z^{*} a$ map

$$
\alpha^{*}\left(Z^{*}, Y, X^{*}\right): H\left(T^{\sharp}\left(Z^{*}, Y\right), X^{*}\right) \rightarrow H\left(Z^{*}, S^{\sharp}\left(Y, X^{*}\right)\right)
$$

by

$$
\alpha^{\sharp}\left(Z^{*}, Y, X^{*}\right)=\alpha^{-1}(X, Y, Z)
$$

Then the function

$$
\alpha^{*}: H\left(T^{*}\left(\mathrm{Z}^{*}, \mathcal{Y}\right), \mathfrak{X}^{*}\right) \rightarrow H\left(\mathrm{Z}^{*}, S^{*}\left(\mathcal{Y}, \mathfrak{X}^{*}\right)\right)
$$

is a natural equivalence, i.e. $\alpha^{*}: T^{*}\left(\mathrm{Z}^{*}, \mathcal{Y}\right) \dashv S^{*}\left(\mathcal{Y}, \mathrm{X}^{*}\right)$. Also $\alpha^{\#}=\alpha$.

The proof of Theorem 4.5 is obvious.

It follows from the duality Theorem 4.5 that for every Theorem A involving a natural equivalence $\alpha: S(x, y) \dashv T(Y, Z)$ a dual Theorem $A^{*}$ may be obtained by applying Theorem A to the natural equivalence $\alpha^{\sharp}: T^{*}\left(Z^{*}, Y\right)$ $\dashv S^{\sharp}\left(\mathcal{Y}, x^{*}\right)$ and then writing the result in terms of the categories $x, y$ and $\mathrm{Z}$, the functors $S$ and $T$ and the natural equivalence $\alpha$, i.e. "reversing all arrows" in the categories $x^{*}$ and $Z^{*}$. It is easily seen that in this sense the Theorems 4.4 and $4.4^{*}$ are the dual of each other.

We now consider functors in more than two variables.

Let

$$
S: x, Q_{1}, \cdots, Q_{m}, \bigotimes_{1}, \cdots, \bigotimes_{n} \rightarrow \mathcal{Z}
$$


be a functor covariant in $X, Q_{1}, \cdots, Q_{m}$ and contravariant in $B_{1}, \cdots, \bigotimes_{n}$ and let

$$
T: Q_{1}, \cdots, Q_{m}, B_{1}, \cdots, Q_{n}, Z \rightarrow X
$$

be a functor contravariant in $Q_{1}, \cdots, Q_{m}$ and covariant in $B_{1}, \cdots, B_{n}, \mathrm{Z}$. Then ([2, Theorem 13.2]) $S$ and $T$ may be considered as functors in two variables as follows. Let $y$ be the cartesian product category (see [2])

$$
y=\left(\prod_{i} a_{i}\right) \times\left(\prod_{j} \otimes_{j}^{*}\right) .
$$

Then $S$ may be considered as a covariant functor

$$
S^{\prime}: x, y \rightarrow z
$$

and $T$ as a functor

$$
T^{\prime}: y, z \rightarrow x
$$

contravariant in $\mathcal{Y}$ and covariant in $Z$. The case of functors in more than two variables thus may be brought back to that of functors in two variables only.

5. The relative case. We shall now consider the case in which the functors $H$ are replaced by other functors.

Definition 5.1. Let $F: \mathfrak{L} \rightarrow \mathfrak{M}$ be a covariant functor and let $Q: x, x \rightarrow \mathfrak{L}$ be a functor contravariant in the first variable and covariant in the second. The functor $Q$ is called a hom-functor rel. $F$ if there exists a natural equivalence

$$
\gamma: H(x, x) \rightarrow F Q(x, x) .
$$

EXAMPLES 5.2.

(a) The functor $H: x, x \rightarrow \mathfrak{M}$ is a hom-functor relative to the identity functor $E: \mathfrak{T} \rightarrow \mathfrak{M}$.

(b) Let $\varrho$ be the category of abelian groups and homomorphisms. Let

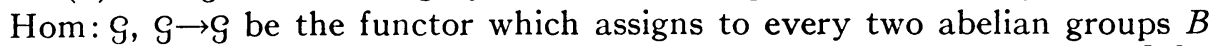
and $C$ the group Hom $(B, C)$ of the homomorphisms of $B$ into $C$ (see [3]). Let $F: \mathcal{G \rightarrow M}$ be the functor which assigns to every group its underlying set. Then Hom: $\mathcal{G}, \mathcal{G} \rightarrow \mathcal{G}$ is a hom-functor rel. $F$.

(c) Let $a$ be the category of topological spaces and continuous maps and let Map: $a, a \rightarrow a$ be the functor which assigns to every two spaces $X$ and $Y$ the function space $\operatorname{Map}(X, Y)=Y^{X}$ with the compact-open topology (see [2]). Let $F: a \rightarrow \mathscr{T}$ be the functor which assigns to every space its underlying set. Then Map: $Q, a \rightarrow Q$ is a hom-functor rel. $F$.

Definition 5.3. Let $S: \mathfrak{X} \rightarrow \mathcal{Z}, T: \mathcal{Z} \rightarrow \mathfrak{X}$ and $F: \mathfrak{L} \rightarrow \mathfrak{N}$ be covariant functors and let $Q: X, X \rightarrow \mathfrak{L}$ and $R: \mathcal{Z}, \mathcal{Z} \rightarrow \mathfrak{L}$ be hom-functors rel. $F$. Let

$$
\beta: R(S(\mathfrak{X}), \mathrm{Z}) \rightarrow Q(\mathscr{X}, T(\mathrm{Z}))
$$


be a natural equivalence. Then $S$ is called the left adjoint of $T$ rel. $F$ under $\beta$ and $T$ the right adjoint of $S$ rel. $F$ under $\beta$ (Notation $\beta: S \dashv T$ rel. $F$ ).

It will now be shown that adjointness rel. $F$ implies adjointness.

TheOREM 5.4. Let $\beta: S \dashv T$ rel. $F$. Then there exists a natural equivalence

$$
\alpha: H(S(X), \mathrm{Z}) \rightarrow H(X, T(\mathrm{Z})),
$$

i.e. $\alpha: S \dashv T$.

Proof. As $Q$ and $R$ are hom-functors rel. $F$ there exist natural equivalences

$$
\begin{aligned}
& \gamma: H(x, \mathfrak{X}) \rightarrow F Q(\mathfrak{X}, \mathfrak{X}), \\
& \delta: H(\mathrm{Z}, \mathrm{Z}) \rightarrow F R(\mathrm{Z}, \mathrm{Z}) .
\end{aligned}
$$

Define for every object $X \in X$ and $Z \in Z$

$$
\alpha(X, Z)=\gamma^{-1}(X, T Z) \circ F \beta(X, Z) \circ \delta(S X, Z) .
$$

Then clearly $\alpha$ is a natural equivalence because $\gamma(x, T(Z)), F \beta(X, Z)$ and $\delta(S(X), Z)$ are so.

We now state the corresponding results for functors in two variables.

Definition 5.5. Let $S: \mathfrak{X}, \mathcal{Y} \rightarrow \mathrm{Z}$ and $F: \mathfrak{L} \rightarrow \mathfrak{M}$ be covariant functors, let $T: Y, Z \rightarrow X$ be a functor contravariant in $Y$ and covariant in $\mathrm{Z}$ and let $Q: X, X \rightarrow \mathcal{L}$ and $R: \mathcal{Z}, \mathcal{Z} \rightarrow \mathscr{L}$ be hom-functors rel. $F$. Let

$$
\beta: R(S(x, y), \mathrm{Z}) \rightarrow Q(x, T(Y, Z))
$$

be a natural equivalence. Then $S$ is called the left adjoint of $\mathfrak{M}$ rel. $F$ under $\beta$ and $T$ the right adjoint of $S$ rel. $F$ under $\beta$ (Notation $\beta: S \dashv T$ rel. $F$ ).

Theorem 5.6. If $\beta: S(X, Y) \dashv T(Y, Z)$ rel. $F$ then there exists a natural equivalence

$$
\alpha: H(S(x, y), z) \rightarrow H(x, T(Y, z)),
$$

i.e. $\alpha: S(x, y) \dashv T(\mathcal{Y}, \mathrm{z})$.

Example 5.7. Let the functors Hom: $\mathcal{G}, \mathcal{G \rightarrow} G$ and $F: \mathcal{G \rightarrow M}$ be as in

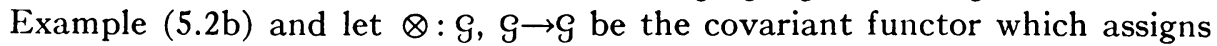
to every two abelian groups $A$ and $B$ their tensor product $A \otimes B$ (see [3]). As is well known (see [2]) there exists for every three abelian groups $A, B$ and $C$ an isomorphism $\beta$ : Hom $(A \otimes B, C) \approx \operatorname{Hom}(A, \operatorname{Hom}(B, C))$ which is natural, i.e. there exists a natural equivalence

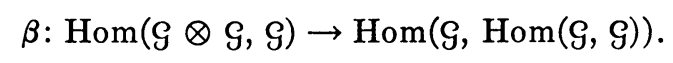

Hence the tensor product $\otimes$ is a left adjoint of the functor Hom (rel. F).

Example 5.8. Let the functors Map: $a, Q \rightarrow a$ and $F: Q \rightarrow \mathfrak{T} C$ be as in Example 5.2c and let $X$ denote the cartesian product. Let $a_{l c}$ be the full subcategory of $a$ generated by the locally compact spaces. As is well known for 
every three spaces $X, Z \in Q$ and $Y \in Q_{l c}$ a homeomorphism $\beta: Z^{X \times Y} \approx\left(Z^{Y}\right)^{X}$ can be defined as follows. Let $f \in Z^{X \times Y}$, i.e. $f: X \times Y \rightarrow Z$ is a continuous map. The map $\beta f: X \rightarrow Z^{Y}$ then maps a point $x \in X$ into the point $(\beta f) x \in Z^{Y}$, i.e. the map $(\beta f) x: Y \rightarrow Z$, given by $((\beta f) x) y=\beta(x, y)$ for every point $y \in Y$. This homeomorphism is natural, i.e. there exists a natural equivalence

$$
\beta: \operatorname{Map}\left(a \times a_{l c}, a\right) \rightarrow \operatorname{Map}\left(a, \operatorname{Map}\left(a_{l c}, a\right)\right) .
$$

Hence the cartesian product $\times$ is a left adjoint of the functor Map (rel. F).

ExAmple 5.9. Let $I$ denote the unit interval. Then it follows from Example 5.8 that the functor $X I: Q \rightarrow Q$ is a left adjoint of the functor Map $(I, \quad)$ : $a \rightarrow Q$, i.e. "taking the cartesian product with the unit interval" is a left adjoint of "taking the space of all paths" (rel. F).

As is "well known" the homotopy relation for continuous maps may be defined using either the functor $X I$ or the functor Map $(I, \quad)$ as follows. Let $P$ be a space consisting of one point $p$ and let $\rho_{0}: P \rightarrow I$ (resp. $\rho_{1}: P \rightarrow I$ ) be the map given by $\rho_{0} p=0$ (resp. $\rho_{1} p=1$ ). For every space $X$ let maps $\phi_{X}: X \rightarrow X \times P$ and $\psi_{X}: \operatorname{Map}(P, X) \rightarrow X$ be defined by $\phi_{X} x=(x, p)$ and $\psi_{X} f=f p$ for every point $x \in X$ and map $f: P \rightarrow X$. Then two maps $f_{0}, f_{1}: X \rightarrow Y \in A$ are homotopic

(i) if there exists a map $g: X \times I \rightarrow Y \in Q$ such that

$$
f_{\epsilon}=g \circ\left(X \times \rho_{\epsilon}\right) \circ \phi_{X} \quad \epsilon=0,1
$$

or equivalently

(ii) if there exists a map $h: X \rightarrow \operatorname{Map}(I, Y) \in Q$ such that

$$
f_{\epsilon}=\psi_{Y} \circ \operatorname{Map}\left(\rho_{\epsilon}, Y\right) \circ h \quad \epsilon=0,1 .
$$

The equivalence of these two definitions is an immediate consequence of the adjointness of the functors $X I$ and $\operatorname{Map}(I, \quad)$.

ExAmple 5.10. Let $a_{0}$ be the category of topological spaces with a base point, i.e. an object of $a_{0}$ is a pair $(X, x)$ where $X \in Q$ and $x \in X$ is a point, while a map $f:(X, x) \rightarrow(Y, y)$ of $Q_{0}$ is a map $f: X \rightarrow Y \in Q$ such that $f x=y$. Let $S: Q_{0} \rightarrow Q_{0}$ be the covariant functor which assigns to every object $(X, x)$ $\in Q_{0}$ its suspension $\left(X^{\prime}, x^{\prime}\right)$ defined as follows. Let $S^{1}$ be a 1 -sphere and let $s \in S^{1}$ be a point. Then $X^{\prime}$ is obtained from $X \times S^{1}$ by shrinking to a point of the subspace $\left(x \times S^{1}\right) \cup(X \times s)$ and $x^{\prime}$ is the image of $(x, s)$ under the identification map $X \times S^{1} \rightarrow X^{\prime}$. Let $\mathrm{Map}_{0}: Q_{0}, Q_{0} \rightarrow a_{0}$ be the functor which assigns to every two objects $(X, x)$ and $(Y, y)$ of $a_{0}$ the pair $\operatorname{Map}_{0}((X, x),(Y, y))$ $=(Z, z)$, where $Z$ is the function space $(Y, y)^{(X, x)}$ (with the compact-open topology) and where the map $z:(X, x) \rightarrow(Y, y)$ is given by $z g=y$ for every point $g \in X$. Let the functor $F: Q_{0} \rightarrow \mathfrak{M}$ assign to every object $(X, x) \in Q_{0}$ the underlying set of the space $X$, then clearly Map ${ }_{0}: a_{0}, Q_{0} \rightarrow Q_{0}$ is a homfunctor rel. $F$. Let $\Omega=\operatorname{Map}_{0}\left(\left(S^{1}, s\right), \quad\right)$, the loop functor. Then analogous to Example 5.8 for every two objects $(X, x),(Y, y) \in Q_{0}$ a homeomorphism $\beta_{0}: \operatorname{Map}_{0}(S(X, x),(Y, y)) \approx \operatorname{Map}_{0}((X, x), \Omega(Y, y))$ can be given which is 
natural, i.e. there exist a natural equivalence

$$
\beta_{0}: \operatorname{Map}_{0}\left(S\left(a_{0}\right), a_{0}\right) \rightarrow \operatorname{Map}_{0}\left(a_{0}, \Omega\left(a_{0}\right)\right) .
$$

Hence the suspension functor $S$ is a left adjoint of the loop functor $\Omega$ (rel. F).

Example 5.11. This example is due to P. J. Hilton. Let $a_{0}$ be the category of topological spaces with a base point (see Example 5.10). Let $X^{2}: a_{0} \rightarrow a_{0}$ be the covariant functor such that for every object $\left(Y, y_{0}\right) \in a_{0}$,

$$
\times^{2}\left(Y, y_{0}\right)=\left(Y \times Y, y_{0} \times y_{0}\right)
$$

and let $V^{2}: a_{0} \rightarrow Q_{0}$ be the covariant functor such that for every object $\left(X, x_{0}\right) \in a_{0}$

$$
\bigvee^{2}\left(X, x_{0}\right)=\left(X \vee X, x_{0} \times x_{0}\right)
$$

where $X \bigvee X=X \times x_{0} \cup x_{0} \times X \subset X \times X$. Let the functor Map $\mathrm{p}_{0}: a_{0}, a_{0} \rightarrow a_{0}$ be as in Example 5.11. Then for every two objects $\left(X, x_{0}\right),\left(Y, y_{0}\right) \in a_{0}$ a homeomorphism $\beta: \operatorname{Map}_{0}\left(\bigvee^{2}\left(X, x_{0}\right),\left(Y, y_{0}\right)\right) \rightarrow \operatorname{Map}_{0}\left(\left(X, x_{0}\right), X^{2}\left(Y, y_{0}\right)\right)$ may be defined by $(\beta f) x=\left(f\left(x \times x_{0}\right) \times f\left(x_{0} \times x\right)\right)$ for every map $f: \bigvee^{2}\left(X, x_{0}\right) \rightarrow\left(Y, y_{0}\right)$ and point $x \in X$. Clearly is natural, i.e. there exists a natural equivalence

$$
\beta: \operatorname{Map}_{0}\left(V^{2}\left(a_{0}\right), a_{0}\right) \rightarrow \operatorname{Map}_{0}\left(a_{0}, X^{2}\left(a_{0}\right)\right) \text {. }
$$

Hence the functor $\bigvee^{2}$ is a left adjoint of the functor $X^{2}$ (rel. $\left.F\right)$.

6. Two natural transformations. Let $S: X \rightarrow Z$ and $T: Z \rightarrow X$ be covariant functors and let $\alpha: S \dashv T$. Then we may define a natural transformation

$$
\kappa: E(x) \rightarrow T S(x)
$$

where $E: X \rightarrow X$ denotes the identity functor, by assigning to every object $X \in X$ the map $\kappa X: X \rightarrow T S X$ given by

$$
\kappa X=\alpha i_{S X} .
$$

It must of course be verified that the function $\kappa$ so defined is natural. It follows from the naturality of $\alpha$ that for every map $x: X \rightarrow X^{\prime} \in X$ commutativity holds in the diagram

$$
\begin{aligned}
& H(S X, S X) \stackrel{\alpha}{\longrightarrow} H(X, T S X) \\
& \downarrow H(S X, S x) \quad \downarrow H(X, T S x) \\
& H\left(S X, S X^{\prime}\right) \stackrel{\alpha}{\longrightarrow} H\left(X, T S X^{\prime}\right) \\
& \uparrow H\left(S x, S X^{\prime}\right) \quad \uparrow H\left(x, T S X^{\prime}\right) \\
& H\left(S X^{\prime}, S X^{\prime}\right) \stackrel{\alpha}{\longrightarrow} H\left(X^{\prime}, T S X^{\prime}\right)
\end{aligned}
$$

Consequently 


$$
\begin{aligned}
T S x \circ \kappa X & =H(X, T S x) \alpha i_{S X}=\alpha H(S X, S x) i_{S X} \\
& =\alpha\left(S x \circ i_{S X}\right)=\alpha\left(i_{S X^{\prime}} \circ S x\right) \\
& =\alpha H\left(S x, S X^{\prime}\right) i_{S X^{\prime}}=H\left(x, T S X^{\prime}\right) \alpha i_{S X^{\prime}}=\kappa X^{\prime} \circ x,
\end{aligned}
$$

i.e. $\kappa$ is natural.

The natural transformation $\kappa$ will be referred to as the natural transformation induced by $\alpha$.

The following lemma expresses the natural equivalence $\alpha$ in terms of the natural transformation $\kappa$. It follows that $\kappa$ completely determines $\alpha$.

Lemma 6.2. Let $\alpha: S(\mathscr{X}) \dashv T(\mathrm{Z})$ and let $\kappa: E(\mathfrak{X}) \rightarrow T S(\mathscr{X})$ be the natural transformation induced by $\alpha$. Then for every object $X \in X$ and $Z \in Z$ and for every map $f: S X \rightarrow Z \in Z$ commutativity holds in the diagram

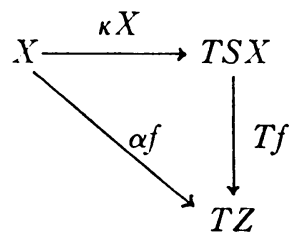

i.e.

$$
\alpha f=T f \circ \kappa X
$$$$
f \in H(S X, Z) .
$$

Proof. It follows from the naturality of $\alpha$ that commutativity holds in the diagram

$$
\begin{aligned}
& H(S X, S X) \stackrel{\alpha}{\longrightarrow} H(X, T S X) \\
& \downarrow H(S X, f) \quad \downarrow H(X, T f) \\
& H(S X, Z) \stackrel{\alpha}{\longrightarrow} H(X, T Z)
\end{aligned}
$$

Consequently

$$
\alpha f=\alpha H(S X, f) i_{S X}=H(X, T f) \alpha i_{S X}=T f \circ \kappa X .
$$

This completes the proof.

Now let $S: X \rightarrow Z$ and $T: \mathcal{Z} \rightarrow \mathscr{X}$ be covariant functors and let $\kappa^{\prime}: E(\mathscr{X})$ $\rightarrow T S(X)$ be a natural transformation. Then $\kappa^{\prime}$ induces a natural transformation

$$
\beta: H(S(x), \mathrm{Z}) \rightarrow H(x, T(\mathrm{Z}))
$$

as follows. For every object $X \in X$ and $Z \in Z$ the $\operatorname{map} \beta: H(S X, Z) \rightarrow H(X, T Z)$ is defined by

$$
\beta f=T f \circ \kappa^{\prime} X \quad f \in H(S X, Z) .
$$


It is readily verified that the function $\beta$ so defined is natural. If $\beta$ is an equivalence for all objects $X \in X$ and $Z \in \mathcal{Z}$, then clearly $\beta: S \dashv T$ and (in view of Lemma 6.2) $\kappa^{\prime}$ is the natural transformation induced by $\beta$. Hence we have:

THEOREM 6.4. Let $S: X \rightarrow Z$ and $T: Z \rightarrow X$ be covariant functors and let $\kappa^{\prime}: E(\mathscr{X}) \rightarrow T S(\mathscr{X})$ be a natural transformation. Then there exists a natural equivalence $\beta: S \dashv T$ which induces $\kappa^{\prime}$ (and hence is unique) if and only if for every object $X \in X$ and $Z \in Z$ the function $\beta: H(S X, Z) \rightarrow H(X, T Z)$ defined by (6.3) is an equivalence.

We shall now dualize the above results.

Let $\alpha: S(\mathscr{X}) \dashv T(\mathrm{Z})$ and let

$$
\kappa^{*}: E\left(\mathrm{Z}^{*}\right) \rightarrow S^{*} T^{*}\left(\mathrm{Z}^{*}\right)
$$

be the natural transformation induced by the natural equivalence $\alpha^{\sharp}$ : $T^{*}\left(Z^{*}\right) \dashv S^{*}\left(x^{*}\right)$. Denote by

$$
\mu: S T(\mathrm{Z}) \rightarrow E(\mathrm{Z})
$$

the natural transformation obtained from $\kappa^{\#}$ by "reversing all arrows" in the categories $x^{*}$ and $Z^{*}$, i.e. for every object $Z \in Z$ the map $\mu Z: S T Z \rightarrow Z$ is given by

$$
\mu Z=\alpha^{-1} i_{T Z} .
$$

The natural transformation $\mu$ will be referred to as the natural transformation induced by $\alpha^{-1}$.

Lemma 6.2*. Let $\alpha: S(\mathfrak{X}) \dashv T(\mathrm{Z})$ and let $\mu: S T(\mathrm{Z}) \rightarrow E(\mathrm{Z})$ be the natural transformation induced by $\alpha^{-1}$. Then for every object $X \in X$ and $Z \in Z$ and for every map $g: X \rightarrow T Z \in Z$ commutativity holds in the diagram

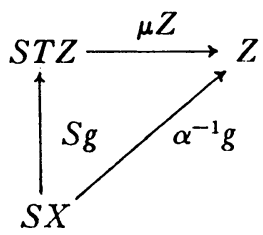

i.e.

$$
\alpha^{-1} g=\mu Z \circ S g
$$$$
g \in H(X, T Z) .
$$

Let $S: \mathbb{X} \rightarrow \mathbb{Z}$ and $T: Z \rightarrow X$ be covariant functors. Then a natural transformation $\mu^{\prime}: S T(\mathrm{Z}) \rightarrow E(\mathrm{Z})$ induces a natural transformation

$$
\gamma: H(x, T(\mathrm{Z})) \rightarrow H(S(x), \mathrm{Z})
$$


and we have:

Theorem 6.4*. Let $S: X \rightarrow Z$ and $T: Z \rightarrow X$ be covariant functors and let $\mu^{\prime}: S T(\mathrm{Z}) \rightarrow E(\mathrm{Z})$ be a natural transformation. Then there exists a natural equivalence $\gamma^{-1}: S \dashv T$ such that $\gamma$ induces $\mu^{\prime}$ (and hence $\gamma$ is unique) if and only if for every object $X \in X$ and $Z \in Z$ the function $\gamma: H(X, T Z) \rightarrow H(S X, Z)$ defined by $6.3^{*}$ is an equivalence.

EXAmple 6.5. Let $Q_{0}$, the category of topological spaces with a base point, the suspension functor $S: \mathfrak{Q}_{0} \rightarrow \mathfrak{Q}_{0}$, the loop functor $\Omega: a_{0} \rightarrow a_{0}$, the hom-functor $\mathrm{Map}_{0}: Q_{0}, Q_{0} \rightarrow Q_{0}$ and the natural equivalence

$$
\beta_{0}: \operatorname{Map}_{0}\left(S\left(Q_{0}\right), Q_{0}\right) \rightarrow \operatorname{Map}_{0}\left(Q_{0}, \Omega\left(Q_{0}\right)\right)
$$

be as in Example 5.10. Using the natural transformation

$$
\kappa: E\left(a_{0}\right) \rightarrow \Omega S\left(a_{0}\right)
$$

induced by $\beta_{0}$ we now define the suspension homomorphism of the homotopy groups (see [4]) and dually using the natural transformation

$$
\mu: S \Omega\left(a_{0}\right) \rightarrow E\left(a_{0}\right)
$$

induced by $\beta_{0}^{-1}$ the suspension homomorphism of the cohomology groups (see [6]) will be obtained.

Let $(Y, y) \in Q_{0}$ and let $S^{n}$ be an $n$-sphere and $s^{n} \in S^{n}$ a point. Clearly $S\left(S^{n}, s^{n}\right) \approx\left(S^{n+1}, s^{n+1}\right)$. As the elements of the $n$th homotopy group $\pi_{n}(Y, y)$ of $(Y, y)$ are the homotopy classes of maps $\left(S^{n}, s^{n}\right) \rightarrow(Y, y)$, i.e. the components of $\operatorname{Map}_{0}\left(\left(S^{n}, s^{n}\right),(Y, y)\right)$, it can easily be verified that the homeomorphism

$$
\beta_{0}: \operatorname{Map}_{0}\left(\left(S^{n+1}, s^{n+1}\right),(Y, y)\right) \approx \operatorname{Map}_{0}\left(\left(S^{n}, s^{n}\right), \Omega(Y, y)\right)
$$

induces an isomorphism

$$
\partial: \pi_{n+1}(Y, y) \approx \pi_{n} \Omega(Y, y) .
$$

The composite homomorphism

$$
\pi_{n}(Y, y) \stackrel{\kappa_{*}}{\longrightarrow} \pi_{n} \Omega S(Y, y) \stackrel{\partial^{-1}}{\longrightarrow} \pi_{n+1} S(Y, y)
$$

now is the suspension homomorphism $\pi_{n}(Y, y) \rightarrow \pi_{n+1} S(Y, y)$.

Let $\pi$ be an abelian group. Then an object $(K, k) \in Q_{0}$ is called of type $(\pi, n)$ if $\pi_{n}(K, k) \approx \pi$ and $\pi_{i}(K, k)=0$ for $i \neq n$. Clearly if $(K, k)$ is of type $(\pi, n)$, then $\Omega(K, k)$ is of type $(\pi, n-1)$. Now let $(K, k)$ be of type $(\pi, n)$ and let $(X, x) \in a_{0}$. If $X$ is "reasonably smooth" then the elements of the $n$th cohomology group $H^{n}(X, x ; \pi)$ of $(X, x)$ with coefficients in $\pi$ are in one-toone correspondence with the homotopy classes of maps $(X, x) \rightarrow(K, k)$, i.e. 
with the components of $\operatorname{Map}_{0}((X, x),(K, k))$. It may then be verified that the homeomorphism

$$
\beta_{0}^{-1}: \operatorname{Map}_{0}((X, x), \Omega(K, k)) \rightarrow \operatorname{Map}_{0}(S(X, x),(K, k))
$$

induces an isomorphism

$$
\delta: H^{n-1}(X, x ; \pi) \approx H^{n}(S(X, x) ; \pi)
$$

and that the composite homomorphism

$$
H^{n}(X, x ; \pi) \stackrel{\mu^{*}}{\longrightarrow} H^{n}(S \Omega(X, x) ; \pi) \stackrel{\delta^{-1}}{\longrightarrow} H^{n-1}(\Omega(X, x) ; \pi)
$$

is the suspension homomorphism $H^{n}(X, x ; \pi) \rightarrow H^{n-1}(\Omega(X, x) ; \pi)$.

Chapter II. Direct and inverse limits

7. Direct limits. Let $Z$ be a category and let $v$ be a proper category (i.e. the objects of $v$ form a set). Let $K: V \rightarrow Z$ be a covariant functor. Then $K$ may be considered as a $V$ diagram over $Z$, i.e. a system of objects and maps of $Z$ indexed by the objects and maps of $v$. We shall now define what we mean by a direct limit of such a system.

Let $Z_{V}$ denote the category of $V$ diagrams over $Z$, i.e. the category of which the objects are the covariant functors $\mathcal{U} \rightarrow \mathcal{Z}$ and of which the maps are the natural transformations between them (see $[2, \S 8]$ ). The category $Z_{V}$ satisfies condition 2.1 because $v$ is proper. Let

$$
E_{V}: \mathrm{Z} \rightarrow \mathrm{Z}_{V}
$$

be the embedding functor which assigns to every object $Z \in Z$ the constant functor $E_{V} Z: V \rightarrow Z$ which maps every object of $v$ into $Z$ and every map into $i_{z}$, and which assigns to every map $z: Z \rightarrow Z^{\prime} \in Z$ the natural transformation $E_{V} z: E_{V} Z \rightarrow E_{V} Z^{\prime}$ given by $\left(E_{V} z\right) V=z$ for every object $V \in v$. We then define

Definition 7.1. Let $A \in \mathrm{Z}$ be an object and let $k: K \rightarrow E_{V} A \in Z_{V}$ be a map. Then $A$ is called the direct limit of $K$ under the map $k$ if for every object $B \in \mathrm{Z}$ and every map $k^{\prime}: K \rightarrow E_{V} B \in \mathrm{Z}_{V}$ there exists a unique map $f: A \rightarrow B \in \mathcal{Z}$ such that commutativity holds in the diagram

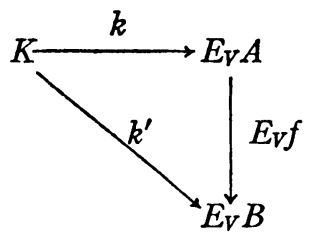

i.e. $E_{V} f \circ k=k^{\prime}\left(\right.$ Notation $\left.A=\lim _{k} K\right)\left({ }^{2}\right)$.

EXAMPLE 7.2. Let $U$ be the category of which the objects are the elements

${ }^{(2)}$ A similar definition of direct limit has, for the case of groups, been given in mimeographed notes of lectures of R. H. Fox (Princeton, 1955). 
of some set $V$ and which has no maps other than identity maps. Let $Q$ be the category of topological spaces. A functor $K: v \rightarrow Q$ (which is both covariant and contravariant) is then merely a collection $\left\{X_{\alpha}\right\}$ of topological spaces indexed by the set $V$. Let $X=\mathrm{U}_{\alpha \in V} X_{\alpha}$ be their union (the points of $X$ are the pairs $(\alpha, x)$ where $\alpha \in V$ and $x \in X)$. For each $\alpha \in V$ let $k_{\alpha}: X_{\alpha} \rightarrow X$ denote the embedding map given by $k_{\alpha} x=(\alpha, x)$ for $x \in X_{\alpha}$. Then $X$ is the direct limit of $K$ under the map $k: K \rightarrow E_{V} X$ defined by $k \alpha=k_{\alpha}$ for all $\alpha \in V$.

EXAMPLE 7.3. Let $G$ be the category of abelian groups and let $v$ be as in Example 7.2. A functor $K: \vartheta \rightarrow \mathcal{G}$ then is a collection $\left\{G_{\alpha}\right\}$ of abelian groups indexed by the set $V$. Let $G=\sum_{\alpha \in V} G_{\alpha}$ be their direct sum (see [3]). For each $\alpha \in V$ let $k_{\alpha}: G_{\alpha} \rightarrow G$ be the injection. Then $G$ is the direct limit of $K$ under the map $k: K \rightarrow E_{V} G$ defined by $k \alpha=k_{\alpha}$ for all $\alpha \in V$.

ExAmple 7.4. Let $D$ be a directed set, i.e. a quasi-ordered set such that for each pair of elements $d_{1}, d_{2} \in D$ there exists a $d_{3} \in D$ such that $d_{1}<d_{3}$ and $d_{2}<d_{3}$. A directed set $D$ may be regarded as a category $D$ (see [2]) of which the objects are the elements of $D$ and which has one map $\left(d_{2}, d_{1}\right): d_{1} \rightarrow d_{2}$ for each pair $\left(d_{2}, d_{1}\right)$ such that $d_{1}<d_{2}$. Enlarge $D$ to a category $D_{\infty}$ by adding one object $\infty$ and for every element $d \in D$, one map $(\infty, d): d \rightarrow \infty$. Then the following definition of direct limit is implicitly contained in [2].

Let $K: D \rightarrow \mathrm{Z}$ be a covariant functor and let the functor $K_{\infty}: D_{\infty} \rightarrow \mathrm{Z}$ be an extension of $K$. Then the object $K_{\infty} \infty \in \mathrm{Z}$ is called the direct limit of $K$ under $K_{\infty}$ if for every extension $K_{\infty}^{\prime}: D \rightarrow Z$ of $K$ there exists a unique natural transformation $\sigma: K_{\infty} \rightarrow K_{\infty}^{\prime}$ such that each $\sigma d$ with $d \neq \infty$ is the identity. It is easily verified that this definition is equivalent with Definition 7.1 for $v=D$.

In general not every object of $Z_{V}$ will have a direct limit (under some map). In order that every object of $Z_{V}$ has a direct limit under some map it is necessary and sufficient that the functor $E_{V}: \mathrm{Z} \rightarrow \mathrm{Z}_{V}$ has a left adjoint. A more precise formulation of both halves of this statement is given in the following two theorems.

THEOREM 7.5. Let $L: Z_{V} \rightarrow Z$ be a covariant functor, let $\alpha: L\left(Z_{V}\right) \dashv E_{V}(\mathrm{Z})$ and let $\kappa: E\left(Z_{V}\right) \rightarrow E_{V} L\left(Z_{V}\right)$ be the natural transformation induced by $\alpha$. Then

$$
L K=\lim _{\kappa} K
$$

for every object $K \in \mathrm{Z}_{V}$.

THEOREM 7.6. Let for every object $K \in Z_{V}$ be given an object $L K \in Z$ and a map $\kappa K: K \rightarrow E_{V} L K \in Z_{V}$ such that $L K=\lim _{\kappa} K$. Then

(i) the function $L$ (defined only for objects of $\mathrm{Z}_{V}$ ) may be extended uniquely to a functor $L_{:} \mathrm{Z}_{V} \rightarrow \mathrm{Z}$ such that the function $\kappa$ becomes a natural transformation $\kappa: E\left(\mathrm{Z}_{V}\right) \rightarrow E_{V} L\left(\mathrm{Z}_{V}\right)$,

(ii) there exists a natural equivalence $\alpha: L\left(Z_{V}\right) \dashv E_{V}(\mathrm{Z})$ such that $\kappa$ is the natural transformation induced by $\alpha$. In view of Lemma $6.2 \alpha$ is unique. 


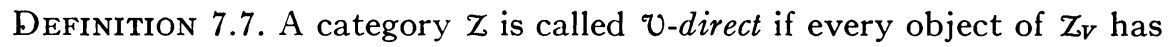
a direct limit under some map.

THEOREM 7.8. A category $\mathrm{Z}$ is $\mathrm{U}$-direct if and only if the functor $E_{V}: \mathrm{Z} \rightarrow \mathrm{Z}_{V}$ has a left adjoint.

REMARK 7.9. The first half of Theorem 7.8 follows directly from Theorem 7.5. In order to obtain the second half of Theorem 7.8 from Theorem 7.6 a kind of axiom of choice would be needed; given for every object of $Z_{V}$ the existence of a direct limit under some map, a choice must be made simultaneously for all objects of $Z_{V}$ (which need not even form a set) of such an object and map. In practice however the statement "every object of $Z_{V}$ has a direct limit under some map" means that it is possible to give a construction which assigns simultaneously to all objects $K \in Z_{V}$ an object $L K \in Z$ and a map $\kappa K: K \rightarrow E_{V} L K$ such that $L K=\lim _{\kappa K} K$. It is in this sense that the notion $V$-direct will be used. The second half of Theorem 7.8 then is an immediate consequence of Theorem 7.6.

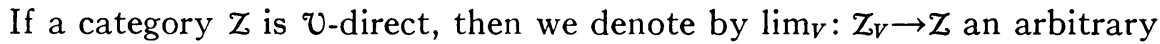
but fixed left adjoint of the functor $E_{V}: \mathcal{Z} \rightarrow \mathcal{Z}_{V}$, by $\alpha_{V}$ an arbitrary but fixed natural equivalence $\alpha_{V}: \lim _{V} \dashv E_{V}$ and by $\lambda_{V}$ the natural transformation induced by $\alpha_{V}$.

Proof of Theorem 7.5. Let $B \in Z$ and $K \in Z_{V}$ be objects. The natural equivalence $\alpha$ yields an equivalence

$$
\alpha: H(L K, B) \rightarrow H\left(K, E_{V} B\right) .
$$

In view of Lemma 6.2 this one-to-one correspondence is given by

$$
\alpha f=E_{V} f \circ \kappa K, \quad f \in H(L K, B),
$$

i.e. for every map $k^{\prime}: K \rightarrow E_{V} B$ there is a unique map $f: L K \rightarrow B$ such that $k^{\prime}=E_{V} f \circ \kappa K$.

Proof of Theorem 7.6. Let $k: K \rightarrow K^{\prime} \in \mathrm{Z}_{V}$ be a map. Then according to Definition 7.1 there exists a unique map $U k: L K \rightarrow L K^{\prime} \in \mathrm{Z}$ such that commutativity holds in the diagram

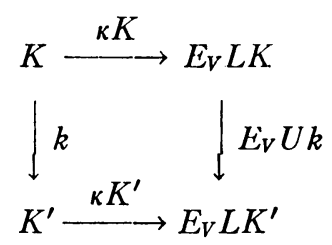

Hence if there exists a functor $L: Z_{V} \rightarrow Z$ with the required property, then it must be defined by $L k=U k$ for every map $k \in Z_{v}$. It is now easily verified that the function $L$ so defined is a covariant functor $L: Z_{V} \rightarrow Z$.

For every object $B \in \mathcal{Z}$ and $K \in \mathcal{Z}_{V}$ define a function 


$$
\alpha: H(L K, B) \rightarrow H\left(K, E_{V} B\right)
$$

by

$$
\alpha f=E_{V} f \circ \kappa K \quad f \in H(L K, B) .
$$

As $L K=\lim _{\kappa K} K$ merely means that $\alpha$ is an equivalence, it follows from Theorem 6.4 that $\alpha$ is a natural equivalence $\alpha: L\left(\mathrm{Z}_{V}\right) \dashv E_{V}(\mathrm{Z})$ such that $\kappa$ is the natural transformation induced by $\alpha$.

8. Inverse limits. The definition of inverse limits and their properties may be obtained from those of direct limits by duality.

Let $x$ be a category, let $\vartheta$ be a proper category and let $K: \vartheta \rightarrow x$ be a contravariant functor. Denote by $K^{\#}: \mathcal{V} \rightarrow \mathfrak{X}^{*}$ the induced covariant functor. Then $K^{\sharp} \in \mathscr{X}_{V}{ }^{*}$. An object $A \in \mathfrak{X}$ then will be called an inverse limit of $K$ if the object $A^{*} \in X^{*}$ is a direct limit of $K^{*}$.

We shall now give the exact definition dual to (7.1).

Let $x^{V}=\left(x_{V}^{*}\right)^{*}$, i.e. $x^{V}$ is the category of the contravariant functors $\mathcal{U} \rightarrow X$ and the natural transformations between them. Let $E_{V}: X^{*} \rightarrow \mathscr{X}_{V}{ }^{*}$ be as in $\S 7$ and let $E^{V}=E_{V}{ }^{*}$, i.e.

$$
E^{V}: x \rightarrow x^{V}
$$

is the embedding functor which assigns to every object $X \in X$ the constant functor $v \rightarrow X$ which maps all of $v$ into $X$ and $i_{X}$.

Definition 8.1. Let $A \in X$ be an object and let $k: E^{V} A \rightarrow K \in X^{V}$ be a map. Then $A$ is called the inverse limit of $K$ under the map $k$ if for every object $B \in X$ and every map $k^{\prime}: E^{v} B \rightarrow K \in X^{v}$ there exists a unique map $f: B \rightarrow A \in X$ such that commutativity holds in the diagram

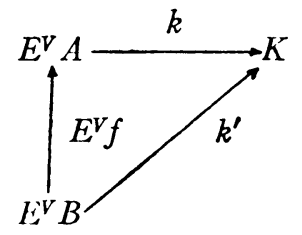

i.c. $k \circ E^{v} f=k^{\prime}\left(\right.$ Notation $\left.A=\lim ^{k} K\right)$.

EXAMPLE 8.2. Let the categories $Q$ and $V$ and the functor $K: V \rightarrow Q$ be as in Example 7.2. Let $Y=\coprod_{\alpha \in V} X_{\alpha}$ be the cartesian product of the spaces $X_{\alpha}$. For every $\alpha \in V$ let $k_{\alpha}: Y \rightarrow X_{\alpha}$ be the projection onto $X_{\alpha}$. Then $Y$ is the inverse limit of $K$ under the map $k: E^{V} Y \rightarrow K$ defined by $k \alpha=k_{\alpha}$ for all $\alpha \in V$.

ExAMPLE 8.3. Let the categories $g$ and $V$ and the functor $K: V \rightarrow G$ be as in Example 7.3. I.et $I I=\coprod_{\alpha \subseteq V} G_{\alpha}$ be the direct product (sec [3]) of the groups $G_{\alpha}$. For each $\alpha \in V$ let $k_{\alpha}: I I \rightarrow G_{\alpha}$ be the projection. Then $I I$ is the inverse limit of $K$ under the map $k: E^{V} I I \rightarrow K$ defined by $k \alpha=k_{\alpha}$ for all $\alpha \in V$.

ExAMrLE 8.4. Let the categories $D$ and $)_{\infty}$ be as in Example 7.4. Then the following definition of inverse limit is implicitly contained in [2]: 
Let $K: D \rightarrow \mathscr{X}$ be a contravariant functor and let the functor $K_{\infty}: D_{\infty} \rightarrow \mathbb{X}$ be an extension of $K$. Then the object $K_{\infty} \infty \in X$ is called the inverse limit of $K$ under $K_{\infty}$ if for every extension $K_{\infty}^{\prime}: D_{\infty} \rightarrow \mathscr{X}$ of $K$ there exists a unique natural transformation $\sigma: K_{\infty}^{\prime} \rightarrow K_{\infty}$ such that $\sigma d$ with $d \neq \infty$ is the identity. It is easily verified that this definition is equivalent with Definition 8.1 for $v=\mathfrak{D}$.

We now dualize Definition 7.7 and Theorem 7.8.

Definition 8.5. A category $X$ is called $v$-inverse if every object of $x^{v}$ has an inverse limit under some map.

Theorem 8.6. A category $x$ is $v$-inverse if and only if the functor $E^{v}$ : $x \rightarrow X^{V}$ has a right adjoint.

If the category $X$ is $\mathcal{V}$-inverse, then we denote by $\lim ^{V}: X^{V} \rightarrow X$ an arbitrary but fixed right adjoint of the functor $E^{V}: X \rightarrow X^{V}$, by $\alpha^{V}$ an arbitrary but fixed natural equivalence $\alpha^{V}: E^{V} \dashv \lim ^{v}$ and by $\lambda^{V}$ the natural transformation induced by $\left(\alpha^{V}\right)^{-1}$.

9. Direct and inverse categories.

Definition 9.1. A category $Z$ is said to have direct limits if it is $U$-direct for every proper category $v$, i.e. if for every proper category $v$ each object of $\mathrm{Z}_{V}$ has a direct limit (under some map).

Examples 9.2. Examples of categories which have direct limits are

(a) the category $\mathfrak{T}$ of sets,

(b) the category 9 of abelian groups and

(c) the category $a$ of topological spaces.

A necessary and sufficient condition in order that a category have direct limits is the existence of a left adjoint of a certain functor. The exact formulation of both halves of this statement will be given in the Theorems 9.4 and 9.5 below which are analogous to the Theorems 7.5 and 7.6.

Let $\mathrm{Z}$ be a category. Define a category $Z_{d}$, the category of all diagrams over $Z$, (a generalization of the category $\mathfrak{D i r}$ of [2]) as follows. An object of $Z_{d}$ is a pair $(\vartheta, K)$ where $\vartheta$ is a proper category and $K: \vartheta \rightarrow Z$ is a covariant functor. Given two objects $(\mathcal{V}, K)$ and $\left(\mathcal{V}^{\prime}, K^{\prime}\right)$ of $\mathcal{Z}_{d}$, a map

$$
(F, k):(\mathcal{V}, K) \rightarrow\left(\mathcal{V}^{\prime}, K^{\prime}\right)
$$

of $\mathrm{Z}_{d}$ is a pair $(F, k)$ where $F$ is a covariant functor

$$
F: v \rightarrow v^{\prime}
$$

and $k$ is a natural transformation

$$
k: K \rightarrow K^{\prime} F
$$

from $K$ to the composite functor $K^{\prime} F: \mathcal{U} \rightarrow \mathbf{Z}$, i.e. for every map $v: V_{1} \rightarrow V_{2} \in \mathcal{V}$ commutativity holds in the diagram 


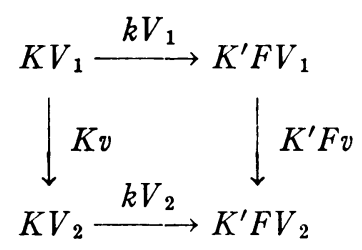

Now let

$$
\left(F^{\prime}, k^{\prime}\right):\left(\mathcal{U}^{\prime}, K^{\prime}\right) \rightarrow\left(\mathcal{V}^{\prime \prime}, K^{\prime \prime}\right)
$$

be another map in $Z_{d}$. Then for every map $v: V_{1} \rightarrow V_{2} \in \mathcal{V}$ commutativity also holds in the diagram

$$
\begin{array}{l|l}
K V_{1} \stackrel{k V_{1}}{\longrightarrow} K^{\prime} F V_{1} \stackrel{k^{\prime} F V_{1}}{\longrightarrow} K^{\prime \prime} F^{\prime} F V_{1} \\
\downarrow K v \quad \downarrow K^{\prime} F v & K^{\prime \prime} F^{\prime} F v \\
K V_{2} \stackrel{k V_{2}}{\longrightarrow} K^{\prime} F V_{2} \stackrel{k^{\prime} F V_{2}}{\longrightarrow} K^{\prime \prime} F^{\prime} F V_{2}
\end{array}
$$

and composition in $Z_{d}$ is defined by

$$
\left(F^{\prime}, k^{\prime}\right) \circ(F, k)=\left(F^{\prime} F, k^{\prime} F \circ k\right) .
$$

It follows immediately from the above diagram that the collection $Z_{d}$ so defined is a category. That $Z_{d}$ satisfies condition 2.1 follows from the fact that only proper categories $\mathcal{V}$ are used.

The effect of fixing the proper caregory $v$ in the object $(V, K)$ is to restrict $Z_{d}$ to the subcategory $Z_{V}$.

Let $\mathcal{O}$ be an arbitrary but fixed category which contains only one object and its identity map. Let

$$
E_{d, 0}: \mathrm{Z}_{0} \rightarrow \mathrm{Z}_{d}
$$

be the inclusion functor. Then we define an embedding functor

$$
E_{d}: \mathrm{Z} \rightarrow \mathrm{Z}_{d}
$$

as the composite functor $E_{d}=E_{d, 0} E_{0}$. Thus $E_{d} A=\left(\mathcal{O}, E_{0} A\right)$ for every object $A \in \mathrm{Z}$.

For every proper category $v$ denote by

$$
O_{V}: v \rightarrow \mathcal{O}
$$

the only such functor (which is both covariant and contravariant).

The following lemma relates the definition of direct limits with the embedding functor $E_{d}: \mathrm{Z} \rightarrow \mathrm{Z}_{d}$.

Lemma 9.3. Let $A \in \mathrm{Z}$ be an object and let $k: K \rightarrow E_{V} A \in \mathrm{Z}_{V}$ be a map. Then $A=\lim _{k} K$ if and only if for every object $B \in \mathrm{Z}$ and every map $\left(O_{V}, k^{\prime}\right):(\mathcal{V}, K)$ 
$\rightarrow E_{d} B \in \mathrm{Z}_{d}$ there exists a unique map $f: A \rightarrow B \in \mathrm{Z}$ such that commutativity holds in the diagram

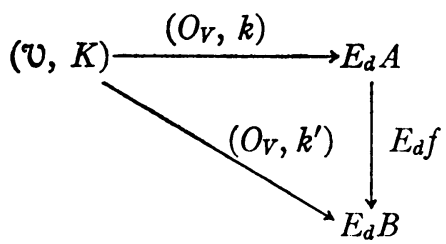

Proof. It is easily verified that $E_{d} f=\left(O_{0}, E_{0} f\right)$ and $\left(E_{0} f\right) O_{V}=E_{V} f$. In view of the definition of composition in $Z_{d}$ commutativity in (9.3a) is equivalent with the condition

$$
\begin{aligned}
O_{V} & =O_{V} O_{0}, \\
k^{\prime} & =\left(E_{0} f\right) O_{V} \circ k=E_{V} \circ \circ k .
\end{aligned}
$$

The first half of this condition is an identity while the second part expresses exactly the condition of Definition 7.1. This proves the lemma.

THEOREM 9.4. Let $L: \mathrm{Z}_{d} \rightarrow \mathrm{Z}$ be a covariant functor, let $\alpha: L\left(\mathrm{Z}_{d}\right) \dashv E_{d}(\mathrm{Z})$ and let $\kappa: E\left(Z_{d}\right) \rightarrow E_{d} L\left(Z_{d}\right)$ be the natural transformation induced by $\alpha$. Then for every object $(\mathcal{V}, K) \in \mathcal{Z}_{d}$,

$$
L(\mathcal{V}, K) \lim _{k} K
$$

where $k$ is given by $\left(O_{V}, k\right)=\kappa(v, K)$.

THEOREM 9.5. Let for every object $(\mathcal{V}, K) \in \mathcal{Z}_{d}$ be given an object $L(\mathcal{V}, K) \in \mathcal{Z}$ and a map $\kappa(\vartheta, K):(\vartheta, K) \rightarrow E_{d} L(\vartheta, K) \in Z_{d}$ such that $L(\vartheta, K)=\lim _{k} K$ where $k$ is defined by $\left(O_{V}, k\right)=\kappa(\mathcal{V}, K)$, then

(i) the function $L$ (defined only for objects of $Z_{d}$ ) may be extended uniquely to a functor $L: Z_{d} \rightarrow Z$ such that the function $\kappa$ becomes a natural transformation $\kappa: E\left(\mathrm{Z}_{d}\right) \rightarrow E_{d} L\left(\mathrm{Z}_{d}\right)$,

(ii) there exists a natural equivalence $\alpha: L\left(Z_{d}\right) \dashv E_{d}(\mathbf{Z})$ such that $\kappa$ is the natural transformation induced by $\alpha$. In view of Lemma $6.2 \alpha$ is unique.

The proofs of these theorems are similar to those of Theorems 7.5 and 7.6; Lemma 9.3 is used instead of Definition 7.1.

The following theorem is analogous to Theorem 7.8. A remark similar to Remark 7.9 applies.

Theorem 9.6. A category $\mathrm{Z}$ has direct limits if and only if the functor $E_{d}: \mathrm{Z} \rightarrow \mathrm{Z}_{d}$ has a left adjoint.

If a category $Z$ has direct limits, then we shall denote by $\lim _{d}: Z_{d} \rightarrow Z$ an arbitrary but fixed left adjoint of the functor $E_{d}: \mathcal{Z} \rightarrow \mathcal{Z}_{d}$, by $\alpha_{d}$ an arbitrary 
but fixed natural equivalence $\alpha_{d}: \lim _{d} \dashv E_{d}$ and by $\lambda_{d}$ the natural transformation induced by $\alpha_{d}$.

We shall now state the corresponding (dual) result of Theorem 9.6 for inverse limits.

Definition $9.1^{*}$. A category $X$ is said to have inverse limits if it is $v$-inverse for every proper category $U$.

ExAmples 9.2*. Examples of categories which have inverse limits are

(a) the category $\mathfrak{T}$ of sets,

(b) the category $\mathcal{G}$ of abelian groups and

(c) the category $a$ of topological spaces.

Let $x^{i}=\left(x_{d}{ }^{*}\right)^{*}$. Consider the functor $E_{d}: X^{*} \rightarrow X_{d}{ }^{*}$ and let $E^{i}=E_{d}{ }^{*}$, i.e.

$$
E^{i}: x \rightarrow x^{i}
$$

is the dual embedding functor.

THEOREM 9.6*. A category $x$ has inverse limits if and only if the functor $E^{i}: X \rightarrow X^{i}$ has a right adjoint.

\section{Chapter III. Existence THEOREMS}

10. Subdivision of a category. With a proper category $v$ we may associate a linear graph $V$ with oriented 1 -simplices, of which the vertices are in one-toone correspondence with the objects of $v$ and of which the 1 -simplices are in one-to-one correspondence with the maps of $V$ which are not an identity; each 1-simplex is oriented from "the vertex of the domain" to "the vertex of the range" of the corresponding map of $\vartheta$. The subdivision of $V$ is a linear graph $V^{\wedge}$ defines as follows. The vertices of $V^{\wedge}$ are the vertices of $V$ and the centers of the 1-simplices of $V$. The 1-simplices of $V^{\wedge}$ are the halves of the 1simplices of $V$, each half being oriented from the center of the original 1-simplex. The subdivision of the category $V$ then is a category for which $V^{\wedge}$ is the associated linear graph.

We shall now give an exact definition.

Definition 10.1. Let $v$ be a proper category. By the subdivision of $v$ we mean a category $\mho^{\wedge}$ defined as follows. The objects of $\mho^{\wedge}$ are in one-to-one correspondence with the maps of $V$; the object corresponding to a map $v \in V$ will be denoted by $v^{\wedge}$. Furthermore $\mathcal{V}^{\wedge}$ contains for every map $v: V_{1} \rightarrow V_{2} \in v$

(i) the identity map $i: v^{\wedge} \rightarrow v^{\wedge}$;

(ii) a map $v^{\prime}: v^{\wedge} \rightarrow i_{V_{1}} \wedge$;

(iii) a map $v^{\prime}: v^{\wedge} \rightarrow i_{v_{2}} \wedge$,

only subject to the condition that for every object $V \in V$

$$
i_{V}{ }^{\prime}=i_{V}{ }^{\prime}=i: i_{V}^{\wedge} \rightarrow i_{V} \wedge .
$$

The category $\mho^{\wedge}$ contains no other maps than these. Composition in $\mho^{\wedge}$ need not be defined as no two nonidentity maps can be composed. Clearly $v^{\wedge}$ is also proper. 
As the orientation of the 1-simplices of the linear graph $V^{\wedge}$ is independent of the orientation of the 1 -simplices of $V$ it follows that the categories $V$ and $v^{*}$ have isomorphic subdivisions. This isomorphism is given by the correspondence

$$
\begin{aligned}
& v^{\wedge} \rightleftarrows v^{* \wedge} \\
& v^{\prime} \rightleftarrows v^{* \prime} \\
& v^{\prime} \rightleftarrows v^{* \prime}
\end{aligned}
$$

for every map $v \in \mho$. The categories $\mho^{\wedge}$ and $v^{* \wedge}$ will be identified under this isomorphism.

EXAMPLE 10.2. Let $g$ be the category of abelian groups and let the functor Hom: $\mathcal{G}, \mathcal{G} \rightarrow \mathcal{G}$ be as in Example 5.2b. Let $V$ be a category consisting of two objects $V_{1}$ and $V_{2}$ and three maps $i_{V_{1}}, i_{V_{2}}$ and $v: V_{1} \rightarrow V_{2}$. Let $A, B: \mathcal{V} \rightarrow \mathcal{G}$ be two covariant functors. Consider the set $H(A, B)$ where $A$ and $B$ are considered as objects of the category $\mathcal{G}_{v}$. An element $s \in H(A, B)$ is a pair of maps $s V_{i} \in$ Hom $\left(A V_{i}, B V_{i}\right)(i=1,2)$ such that commutativity holds in the diagram

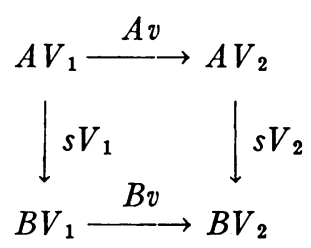

For every two elements $s, t \in H(A, B)$ their sum is defined by

$$
(s+t) V_{i}=s V_{i}+t V_{i}
$$$$
i=1,2 .
$$

This addition converts the set $H(A, B)$ into an abelian group $G$.

In this definition of the object $G \in \mathcal{G}$ use was made of the fact that the functor Hom has its values in the category of abelian groups. Hence in its present form this definition cannot be applied to functors which have their values in another category. In order to overcome this difficulty we shall now show how the object $G \in \mathcal{G}$ may be obtained using only the following two properties of the functor Hom

(i) the functor Hom is contravariant in the first variable and covariant in the second and

(ii) the functor Hom has its values in a category which is $\mho^{\wedge}$-inverse.

Clearly such a definition can be applied to other categories as well.

Consider the diagrams

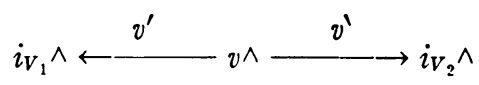

and 
(10.2b) Hom $\left(A V_{1}, J V_{1}\right) \stackrel{\operatorname{Hom}\left(A V_{1}, B v\right)}{\longrightarrow} \operatorname{Hom}\left(A V_{1}, B V_{2}\right) \stackrel{\operatorname{Hom}\left(A v, B V_{2}\right)}{\longleftarrow} \operatorname{Hom}\left(A V_{2}, B V_{2}\right)$ and let $C: v^{\wedge} \rightarrow \mathcal{G}$ be the contravariant functor which assigns to every object or map of $10.2 \mathrm{a}$ the group or homomorphism of $10.2 \mathrm{~b}$ straight underneath. Now $s \in G$ if and only if

$$
B v \circ s V_{1}=s V_{2} \circ A v
$$

or equivalently if and only if

$$
\operatorname{Hom}\left(A V_{1}, B v\right) s V_{1}=\operatorname{Hom}\left(A v, B V_{2}\right) s V_{2} .
$$

It is readily verified that this exactly means that $G$ is an inverse limit of the object $C \in \mathcal{G}^{W}$, where $\mathscr{W}=\mathcal{V}^{\wedge}$. Hence $G$ may be defined in terms of the functors $A, B$ and Hom and inverse limits only.

11. Lifted functors. Following [2] we shall now describe a procedure of obtaining new functors from a given one.

Let $F: \nVdash \rightarrow \&$ be covariant functor and let $\mathcal{V}$ be a proper category. Then the functor $F$ induces a covariant functor

$$
F_{V}: \mathfrak{K}_{V} \rightarrow \mathfrak{L}_{V}
$$

called lifted. The definition of this lifted functor may be described by the following pair of diagrams

$$
\begin{gathered}
V_{1} \stackrel{v}{\longrightarrow} V_{2} \\
F\left(A V_{1}\right) \stackrel{F(A v)}{\longrightarrow} F\left(A V_{2}\right) \\
\downarrow F\left(a V_{1}\right) \quad \mid F\left(a V_{2}\right) \\
F\left(A^{\prime} V_{1}\right) \stackrel{F\left(A^{\prime} v\right)}{\longrightarrow} F\left(A^{\prime} V_{2}\right)
\end{gathered}
$$

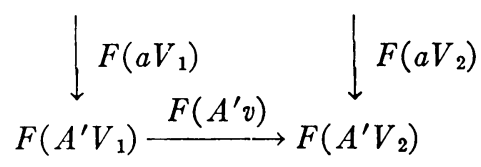

where $v: V_{1} \rightarrow V_{2} \in \mathcal{V}$ and $a: A \rightarrow A^{\prime} \in K_{V}$ are maps. The meaning of these diagrams is that for every object $A \in \mathcal{K}_{V}$ the object $F_{V} A \in \mathfrak{L}_{V}$ is the covariant functor $F_{V} A: \vartheta \rightarrow \mathscr{L}$ which assigns (for every map $v: V_{1} \rightarrow V_{2} \in \mathcal{V}$ ) to the objects and maps of (11.1a) the corresponding objects and maps in the first row of $(11.1 \mathrm{~b})$ and that for every map $a: A \rightarrow A^{\prime} \in \mathcal{K}_{V}$ the map $F_{V} a \in \mathcal{L}_{V}$ is the natural transformation which assigns to the objects of (11.1a) the corresponding "vertical maps" of (11.1b).

Replacing $\vartheta$ by its dual we obtain a lifted functor

$$
F^{v}: \mathfrak{K}^{v} \rightarrow \mathfrak{L}^{V} \text {. }
$$

Similarly for a contravariant functor $F: \mathfrak{K} \rightarrow \mathfrak{L}$ the contravariant lifted functors 


$$
\begin{aligned}
& F_{V}: \mathfrak{K}^{V} \rightarrow \mathfrak{L}_{V}, \\
& F^{V}: \mathfrak{K}_{V} \rightarrow \mathfrak{L}^{V}
\end{aligned}
$$

may be obtained.

By the argument used in $\$ 4$ this may be generalized to functors involving additional variables. The lifted functors then involve the same extra variables with the same variance. For instance a functor $T: \mathcal{Y}, \mathcal{Z} \rightarrow X$ contravariant in $\mathcal{Y}$ and covariant in $\mathrm{Z}$ induces a lifted functor

$$
T^{v}: \mathcal{Y}_{v}, \mathrm{Z} \rightarrow \mathfrak{X}^{v}
$$

contravariant in $\mathcal{Y}_{V}$ and covariant in $\mathrm{Z}$, which is defined by the diagrams

$$
\begin{gathered}
V_{1} \stackrel{v}{\longrightarrow} V_{2} \\
T\left(L V_{1}, Z\right) \stackrel{T(L v, Z)}{\stackrel{ }{\downarrow} T\left(l V_{1}, z\right)} T\left(L V_{2}, Z\right) \\
T\left(L^{\prime} V_{1}, Z^{\prime}\right) \stackrel{T\left(L^{\prime} v, Z^{\prime}\right)}{\longleftarrow} T\left(L^{\prime} V_{2}, Z^{\prime}\right)
\end{gathered}
$$

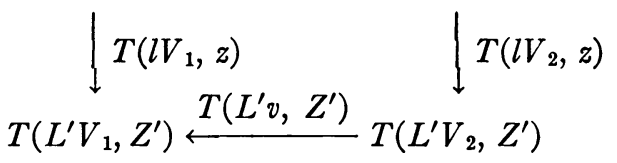

where $v: V_{1} \rightarrow V_{2} \in v, l: L^{\prime} \rightarrow L \in \mathcal{Y}_{V}$ and $z: Z \rightarrow Z^{\prime} \in \mathcal{Z}$ are maps.

Notational convention 11.3. A lifted functor will always have the same additional index in the same position as its range category. This will also apply to the lifted functors defined below.

In the lifted functors defined above only one variable was lifted. These functors will be referred to as lifted in one variable.

For a functor in two variables we shall now define functors which are lifted in two variables simultaneously. Use will be made of the notion of subdivision of a category.

Let $S: \mathfrak{X}, \mathcal{Y} \rightarrow \mathrm{Z}$ be a covariant functor, let $\vartheta$ be a proper category and let $W=\mathcal{V}^{\wedge}$. Then a (covariant) lifted functor

$$
S_{W}: x^{V}, y_{V} \rightarrow \mathrm{Z}_{W}
$$

is defined by the diagrams

$$
i_{V_{1}} \wedge \stackrel{v^{\prime}}{\longleftarrow} v \stackrel{v^{\prime}}{\longrightarrow} i_{V_{2}} \wedge
$$

$$
\begin{aligned}
& S\left(M V_{1}, L V_{1}\right) \stackrel{S\left(M v, L V_{1}\right)}{\longleftarrow} S\left(M V_{2}, L V_{1}\right) \stackrel{S\left(M V_{2}, L v\right)}{\longrightarrow} S\left(M V_{2}, L V_{2}\right) \\
& \text { (11.4b) } \downarrow S\left(m V_{1}, l V_{1}\right) \\
& \downarrow S\left(m V_{2}, l V_{1}\right) \quad \downarrow S\left(m V_{2}, l V_{2}\right) \\
& S\left(M^{\prime} V_{1}, L^{\prime} V_{1}\right) \stackrel{S\left(M^{\prime} v, L^{\prime} V_{1}\right)}{\longleftarrow} S\left(M^{\prime} V_{2}, L^{\prime} V_{1}\right) \stackrel{S\left(M^{\prime} V_{2}, L^{\prime} v\right)}{\longrightarrow} S\left(M^{\prime} V_{2}, L^{\prime} V_{2}\right)
\end{aligned}
$$


where $v: V_{1} \rightarrow V_{2} \in \mathcal{V}, m: M \rightarrow M^{\prime} \in \mathscr{X}^{v}$ and $l: L \rightarrow L^{\prime} \in \mathcal{Y}_{V}$ are maps.

Replacing $\mho$ by its dual we obtain (because $W=V^{\wedge}=V^{* \wedge}$ ) another covariant lifted functor

$$
S_{W}: x_{V}, y^{V} \rightarrow Z_{W} .
$$

Similarly a functor $T: \mathcal{Y}, Z \rightarrow X$, contravariant in $\mathcal{Y}$ and covariant in $\mathcal{Z}$ induces lifted functors

$$
\begin{aligned}
& T^{W}: \mathcal{Y}_{V}, Z_{v} \rightarrow x^{W}, \\
& T^{W}: Y^{V}, z^{V} \rightarrow x^{W}
\end{aligned}
$$

both contravariant in the first variable and covariant in the second.

EXAMPLE 11.5. Let $G$ be the category of abelian groups, let the functor

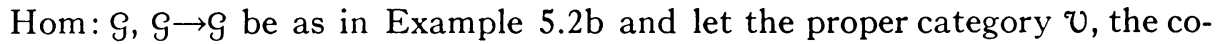
variant functors $A, B: \vartheta \rightarrow \mathcal{G}$ and the contravariant functor $C: \vartheta \rightarrow \mathcal{G}$ be as in Example 10.2. Let $W=\mathcal{V}^{\wedge}$. Then it is readily verified that $C=\operatorname{Hom}^{W}(A, B)$.

12. Existence theorems. Sufficient conditions will be given in order that a functor lifted in one variable has a left or right adjoint. The theorems stated are special cases of the corresponding theorems for the relative case which will be obtained in $\$ 13$.

Let $\alpha: S(X) \dashv T(Z)$. Then for every proper category $v$ the lifted functor $T_{V}: Z_{V} \rightarrow X_{V}$ has a left adjoint and the lifted functor $S_{V}: X_{V} \rightarrow Z_{V}$ has a right adjoint, in fact we have:

TheOREM 12.1. Let $\alpha: S(X) \dashv T(\mathrm{Z})$ and let $v$ be a proper category. Then there exists a natural equivalence

$$
\alpha^{\prime}: H\left(S_{V}\left(\mathfrak{X}_{V}\right), \mathrm{Z}_{V}\right) \rightarrow H\left(\mathfrak{X}_{V}, T_{V}\left(\mathrm{Z}_{V}\right)\right)
$$

i.e.

$$
\alpha^{\prime}: S_{V}\left(\Upsilon_{V}\right) \dashv T_{V}\left(\mathrm{Z}_{V}\right) .
$$

Let $x$ be a $\mathcal{u}$-direct category and let $\vartheta$ be a proper category. Let $g:\left(x_{U}\right)_{V}$ $\rightarrow\left(\mathscr{X}_{V}\right)_{U}$ be the isomorphism which assigns to every object $K \in\left(\mathscr{X}_{U}\right)_{V}$ the object $g K \in\left(x_{V}\right)_{U}$ given by $((g K) U) V=(K V) U$ for every object $U \in \mathcal{U}$ and $V \in v$. Let $E_{U}: X \rightarrow X_{U}$ be the embedding functor used in the definition of direct limit. Compose the functor $\mathscr{g}$ with the lifted functor $\left(E_{U}\right)_{V}$. Then it is readily verified that the composite functor $\mathfrak{g}\left(E_{U}\right)_{V}: X_{V} \rightarrow\left(X_{V}\right)_{U}$ is also such an embedding functor. Hence application of the Theorems 7.8 and 12.1 yields

Corollary 12.2. Let $x$ be a category and let $u$ and $\tilde{v}$ oe proper categories. If $X$ is $\mathcal{U}$-direct, then so is $x_{V}$.

Theorem 12.1 may be generalized to functors in two variables as follows.

Theorem 12.3. Let $\alpha: S(\mathscr{X}, \mathcal{Y}) \dashv T(Y, Z)$ and let $v$ be a proper category. Then there exists a natural equivalence 


$$
\alpha^{\prime}: H\left(S_{V}\left(\mathfrak{x}_{V}, \mathcal{Y}\right), \mathfrak{Z}_{V}\right) \rightarrow H\left(\mathfrak{X}_{V}, T_{V}\left(\mathcal{Y}, \mathfrak{Z}_{V}\right)\right)
$$

i.e. $\alpha^{\prime}: S_{V}\left(\mathfrak{X}_{V}, \mathcal{Y}\right) \dashv T_{V}\left(\mathcal{Y}, \mathfrak{Z}_{V}\right)$.

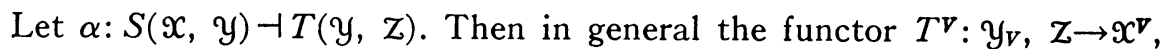
lifted in the variable $\mathcal{Y}$ has no left adjoint. This is however the case if the category $\mathcal{Z}$ is $\mathcal{U}^{\wedge}$-direct. In fact we have

THEOREM 12.4. Let $\alpha: S(x, Y) \dashv T(Y, Z)$, let $v$ be a proper category and let $W=V^{\wedge}$. If the category $\mathcal{Z}$ is $W$-direct, then there exists a natural equivalence

$$
\alpha_{0}: H\left(\lim _{W} S_{W}\left(x^{V}, Y_{V}\right), \mathrm{z}\right) \rightarrow H\left(x^{V}, T^{V}\left(Y_{V}, \mathrm{Z}\right)\right)
$$

i.e. $\alpha_{0}: \lim _{W} S_{W}\left(x^{V}, \mathcal{Y}_{V}\right) \dashv T^{v}\left(\mathcal{Y}_{V}, \mathrm{Z}\right)$.

And by duality

TheOREM 12.4*. Let $\alpha: S(X, Y) \vdash T(Y, Z)$, let $v$ be a proper category and let $W=V^{\wedge}$. If the category $X$ is $W$-inverse, then there exists a natural equivalence

$$
\alpha^{0}: H\left(S_{V}\left(x, Y_{V}\right), Z_{V}\right) \rightarrow H\left(x, \lim ^{W} T^{W}\left(\mathcal{Y}_{V}, Z_{V}\right)\right)
$$

i.e. $\alpha_{0}: S_{V}\left(x, \mathcal{Y}_{V}\right) \dashv \lim ^{W} T^{W}\left(\mathcal{Y}_{V}, Z_{V}\right)$.

The Theorems $12.1,12.3$ and 12.4 follow immediately from the analogous theorems for the relative case $(13.4,13.5$ and 13.8$)$ by putting

$$
\begin{aligned}
& \mathfrak{L}=\mathfrak{M}, \\
& F=E: \mathfrak{T} \rightarrow \mathfrak{M} \text {, the identity functor, } \\
& Q=H: x, x \rightarrow \mathfrak{T}, \\
& R=H: \mathrm{Z}, \mathrm{Z} \rightarrow \mathfrak{I} \text {. }
\end{aligned}
$$

13. The relative case. We shall now extend the existence theorems of $\$ 12$ to the relative case.

Definition 13.1. Let $W$ be a proper category. A covariant functor $F: \mathfrak{L} \rightarrow \mathfrak{M}$ will be called $\mathfrak{W}$-inverse if

(i) $\mathscr{L}$ is $W$-inverse;

(ii) $\Re$ is $\mathscr{W}$-inverse;

(iii) $F$ commutes with inverse limits, i.e. there exists a natural equivalence

$$
\chi: F \lim ^{W}\left(\mathfrak{L}^{W}\right) \rightarrow \lim ^{W} F^{W}\left(\mathfrak{L}^{W}\right)
$$

such that commutativity holds in the diagram

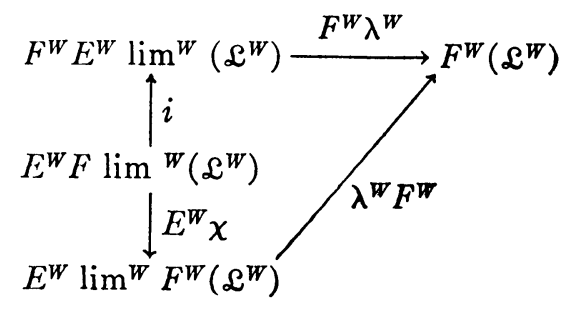


EXAMPLES 13.2. Examples of functors which are $W$-inverse for every proper category $W$ are

(a) the identity functor $E: \mathfrak{M} \rightarrow \mathfrak{M}$,

(b) the functor $F: \mathcal{G \rightarrow T C}$ (see Example 5.2b) which assigns to every abelian group its underlying set, and

(c) the functor $F: Q \rightarrow \mathfrak{T C}$ (see Example 5.2c) which assigns to every topological space its underlying set.

Lemma 13.3. Let $Q: X, X \rightarrow \mathfrak{L}$ be a hom-functor rel. $F$, let $v$ be a proper category and let $W=\mathcal{V}^{\wedge}$. If the functor $F: \mathfrak{L} \rightarrow \mathfrak{W}$ is $W$-inverse, then there exists a natural equivalence

$$
\gamma^{\prime}: H\left(x_{V}, x_{V}\right) \rightarrow F \lim ^{W} Q^{W}\left(x_{V}, x_{V}\right)
$$

i.e. $\lim ^{W} Q^{W}: \mathfrak{X}_{V}, \mathfrak{X}_{V} \rightarrow \mathcal{L}$ is also a hom-functor rel. $F$.

Theorem 13.4. Let $\beta: S(X) \dashv T(Z)$ rel. $F$, let $v$ be a proper category and let $\mathscr{W}=\mathcal{V}^{\wedge}$. If the functor $F: \mathfrak{L} \rightarrow \mathfrak{T}$ is $\mathscr{W}$-inverse, then there exists a natural equivalence

$$
\beta^{\prime}: \lim ^{W} R^{W}\left(S_{V}\left(x_{V}\right), Z_{V}\right) \rightarrow \lim ^{W} Q^{W}\left(x_{V}, T_{V}\left(Z_{V}\right)\right)
$$

i.e., in view of Lemma 13.3, $\beta^{\prime}: S_{V}\left(\mathscr{X}_{V}\right) \dashv T_{V}\left(\mathrm{Z}_{V}\right)$ rel. $F$.

THEOREM 13.5. Let $\beta: S(X, Y) \dashv T(\mathcal{Y}, Z)$ rel. $F$, let $\mathcal{Y}$ be a proper category and let $\mathscr{W}=\mathcal{V}^{\wedge}$. If the functor $F: \mathcal{L} \rightarrow \mathfrak{T}$ is $\mathscr{W}$-inverse, then there exists a natural equivalence

$$
\beta^{\prime}: \lim ^{W} R^{W}\left(S_{V}\left(\mathfrak{X}_{V}, \mathcal{Y}\right), \mathrm{Z}_{V}\right) \rightarrow \lim ^{W} Q^{W}\left(\mathfrak{X}_{V}, T_{V}\left(\mathcal{Y}, \mathrm{Z}_{V}\right)\right)
$$

i.e., in view of Lemma 13.3, $\beta^{\prime}: S_{V}\left(\mathscr{X}_{V}, \mathcal{Y}\right) \dashv T_{V}\left(\mathcal{Y}, \mathrm{Z}_{V}\right)$ rel. $F$.

Definition 13.6. A covariant functor $F: \mathfrak{L} \rightarrow \mathfrak{N}$ will be called true if " $F l$ is an equivalence" implies " $l$ is an equivalence."

ExAmples 13.7. Examples of a true functor are:

(a) the identity functor $E: \mathfrak{M} \rightarrow \mathfrak{M}$;

(b) the functor $F: \mathcal{G} \rightarrow \mathfrak{M}$ (see Example 5.2b) which assigns to every abelian group its underlying set.

The functor $F: Q \rightarrow \mathfrak{N}$ (see Example 5.2c) which assigns to every topological space its underlying set is not true.

Theorem 13.8. Let $\beta: S(X, Y) \dashv T(Y, Z)$ rel. $F$, let $v$ be a proper category and let $W=\mathcal{V}^{\wedge}$. If the functor $F: \mathfrak{L} \rightarrow \mathfrak{M}$ is true and $W$-intverse and the category $\mathrm{Z}$ is $W$-direct, then there exists a natural equivalence

$$
\beta_{0}: R\left(\lim _{W} S_{W}\left(x^{V}, Y_{V}\right), \mathrm{Z}\right) \rightarrow \lim ^{W} Q^{W}\left(\mathfrak{X}^{V}, T^{v}\left(\mathcal{Y}_{V}, \mathrm{Z}\right)\right),
$$

i.e. $\beta_{0}: \lim _{W} S_{W}\left(\mathfrak{X}^{V}, \mathcal{Y}_{V}\right) \dashv T^{v}\left(\mathcal{Y}_{V}, \mathrm{Z}\right)$ rel. $F$.

Theorem 13.8*. Let $\beta: S(X, Y) \dashv T(\mathcal{Y}, Z)$ rel. $F$, let $v$ be a proper category and let $W=\mathcal{V}^{\wedge}$. If the functor $F: \mathfrak{L} \rightarrow \mathfrak{M}$ is true and $\mathscr{W}$-inverse and the category 
$X$ is $W$-inverse, then there exists a natural equivalence

$$
\beta^{0}: \lim ^{W} R^{W}\left(S_{V},\left(x, y_{V}\right), z_{V}\right) \rightarrow Q\left(x, \lim ^{W} T^{W}\left(y_{V}, z_{V}\right)\right)
$$

i.e. $\beta^{0}: S_{V}\left(\mathcal{X}, \mathcal{Y}_{V}\right) \dashv \lim ^{W} T^{W}\left(\mathcal{Y}_{V}, \mathrm{Z}_{V}\right)$ rel. $F$.

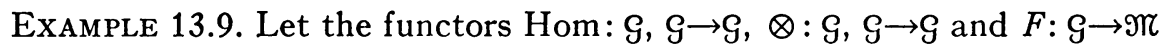
and the natural equivalence

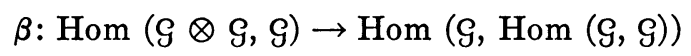

be as in Example 5.7. Let $V$ be a multiplicative system with unit element. Each element $v \in V$ gives rise to a transformation $v: V \rightarrow V$ defined by $v(x)$ $=v x$. Let $v$ denote the proper category which has one object $V$ and has the transformations $v$ as maps. Then $\mathcal{G}_{V}\left(\right.$ resp. $\left.\mathcal{G}^{V}\right)$ is the category of abelian groups with $V$ as left (resp. right) operators. Let $W=V^{\wedge}$, then the category $G$ is both $W$-direct and $W$-inverse and the functor $F$ is $W$-inverse. The functor $F$ is also true. Hence we may apply Theorems $13.5,13.8$ and $13.8^{*}$.

It is readily verified with comparison with the usual definitions (see [1]) that

(i) the functor $\otimes_{V}: \mathcal{G}_{V}, \mathcal{G} \rightarrow \mathcal{G}_{V}$ assigns to every group with operators $A \in \mathcal{G}_{V}$ and every group $B \in \mathcal{G}$ their tensor product $A \otimes B$ with operators induced by those of $A$,

(ii) the functor $\otimes_{V}: \mathcal{G}, \mathcal{G}_{V} \rightarrow \mathcal{G}_{V}$ assigns to every group $A \in \mathcal{G}$ and every group with operators $B \in \mathcal{G V}_{v}$ their tensor product $A \otimes B$ with operators induced by those of $B$,

(iii) the functor $\lim _{W} \otimes_{W}: \mathcal{G}^{V}, \mathcal{G}_{V} \rightarrow \mathcal{G}$ assigns to every right- $V$-group $A \in \mathcal{G}^{V}$ and every left- $V$-group $B \in \mathcal{G}_{V}$ their tensor product $A \otimes_{V} B$ over $V$,

(iv) the functor $\operatorname{Hom}_{V}: \mathcal{G}, \mathcal{G}_{V} \rightarrow \mathcal{G}_{V}$ assigns to every group $A \in \mathcal{G}$ and group with operators $B \in G_{V}$ the group $\operatorname{Hom}(A, B)$ with operators induced by those of $B$,

(v) the functor $\operatorname{Hom}^{v}: \varrho_{v}, \varrho \rightarrow \mathcal{G}^{v}$ assigns to every group with operators $A \in \mathcal{G V}_{V}$ and every group $B \in \mathcal{G}$ the group $\operatorname{Hom}(A, B)$ with operators induced by those of $A$,

(vi) the functor $\lim ^{w} \mathrm{Hom}^{w}: \mathcal{G}_{v}, \mathcal{G}_{v} \rightarrow \mathcal{G}$ assigns to every two groups with operators $A, B \in \mathcal{G}_{V}$ the group $\operatorname{Hom}_{V}(A, B)$ of equivariant homomorphisms $A \rightarrow B$, and

(vii) the functor $\lim ^{W} \operatorname{Hom}^{W}: \mathrm{g}^{V}, \mathrm{~g}^{V} \rightarrow \mathrm{g}$ assigns to every two groups with operators $A, B \in \mathcal{G}^{V}$ the group $\operatorname{Hom}_{V}(A, B)$ of equivariant homomorphisms $A \rightarrow B$.

Application of Theorems $13.5,13.8$ and $13.8^{*}$ thus yields that there exist natural equivalences

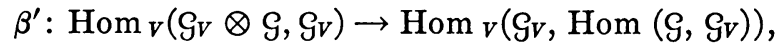

$$
\begin{aligned}
& \beta_{0}: \operatorname{Hom}\left(\mathcal{G}^{V} \otimes_{V} \mathcal{G}_{V}, \mathcal{G}\right) \rightarrow \operatorname{Hom}_{V}\left(\mathcal{G}^{V}, \operatorname{Hom}\left(\mathcal{G}_{V}, \mathcal{G}\right)\right) \text {, } \\
& \beta^{0}: \operatorname{Hom}_{V}\left(\mathcal{G} \otimes \mathcal{G}_{v}, \mathcal{G}_{v}\right) \rightarrow \operatorname{Hom}\left(\mathcal{G}, \operatorname{Hom}_{V}\left(\mathcal{G}_{v}, \mathcal{G}_{v}\right)\right) \text {, }
\end{aligned}
$$


i.e.

$$
\begin{aligned}
& \beta^{\prime}: \mathcal{G}_{V} \otimes \mathcal{G} \dashv \text { Hom }\left(\mathcal{G}, \mathcal{G}_{V}\right) \text { rel. } F \text {., } \\
& \beta_{0}: \mathcal{G}^{V} \otimes_{V} \mathcal{S}_{V} \dashv \text { Hom }\left(\mathcal{G}_{V}, \mathcal{G}\right) \text { rel. } F \text {., } \\
& \beta^{0}: \mathcal{G} \otimes \mathcal{G}_{V} \dashv \operatorname{Hom}_{V}\left(\mathcal{G}_{V}, \mathcal{G}_{V}\right) \text { rel. } F \text {. }
\end{aligned}
$$

Proof of Lemma 13.3. We first consider the case where $\mathscr{L}=\mathfrak{M}, \quad F$ $=E: \mathfrak{N} \rightarrow \mathfrak{N}$, the identity functor and $Q=H: \mathfrak{X}, \mathfrak{X} \rightarrow \mathfrak{M}$. Let $K, K^{\prime} \in \mathfrak{X}_{V}$ be objects. An element $f \in H\left(K, K^{\prime}\right)$ is a function which assigns to every object $V \in \mathcal{V}$ a map $f V \in H\left(K V, K^{\prime} V\right)$ such that for every map $v: V_{1} \rightarrow V_{2} \in V$ commutativity holds in the diagram

or equivalently

$$
\begin{gathered}
K V_{1} \stackrel{K v}{\longrightarrow} K V_{2} \\
\downarrow \downarrow f V_{1} \quad \mid f V_{2} \\
K^{\prime} V_{1} \stackrel{K^{\prime} v}{\longrightarrow} K^{\prime} V_{2}
\end{gathered}
$$

$$
H\left(K v, K^{\prime} V_{2}\right) f V_{2}=H\left(K V_{1}, K^{\prime} v\right) f V_{1} .
$$

Hence $f$ assigns to every map $v: V_{1} \rightarrow V_{2} \in \mathcal{V}$ an element

$$
\left(\gamma^{\prime \prime} f\right) v \wedge=H\left(K v, K^{\prime} V_{2}\right) f V_{2} \in H\left(K V_{1}, K^{\prime} V_{2}\right)
$$

such that

$$
H\left(K v, K^{\prime} V_{2}\right)\left(\gamma^{\prime \prime} f\right) \hat{i_{V_{2}}}=H\left(K V_{1}, K^{\prime} v\right)\left(\gamma^{\prime \prime} f\right) \hat{i_{V_{1}}}=\left(\gamma^{\prime \prime} f\right) v \wedge
$$

i.e. $f$ determines an element $\gamma^{\prime \prime} f \in \lim ^{w} H^{w}\left(K, K^{\prime}\right)$. Straightforward computation now yields that the function

$$
\gamma^{\prime \prime}: H\left(x_{V}, x_{V}\right) \rightarrow \lim ^{w} H^{w}\left(x_{V}, x_{V}\right)
$$

so defined in a natural equivalence.

Because $Q$ is a hom-functor rel. $F$ there exists a natural equivalence.

$$
\gamma: H(x, x) \rightarrow F Q(x, x) .
$$

This induces a natural equivalence

$$
\gamma^{W}: H^{W}\left(x_{V}, x_{V}\right) \rightarrow F^{W} Q^{W}\left(x_{V}, x_{V}\right)
$$

of the lifted functors, given by $\gamma^{W}\left(K, K^{\prime}\right) v^{\wedge}=\gamma\left(K V_{1}, K^{\prime} V_{2}\right)$ for every object $K, K^{\prime} \in \mathscr{X}_{V}$ and every map $v: V_{1} \rightarrow V_{2} \in \mathcal{V}$. Composition of the natural equivalence $\chi\left(F\right.$ is $W$-inverse) with the lifted functor $Q^{W}\left(x_{V}, x_{V}\right)$ yields a natural equivalence

$$
\chi Q^{W}: F \lim ^{W} Q^{W}\left(x_{V}, x_{V}\right) \rightarrow \lim ^{W} F^{W} Q^{W}\left(x_{V}, x_{V}\right) .
$$

The composite natural equivalence 


$$
\gamma^{\prime}=\left(x Q^{W}\right)^{-1} \circ \lim ^{W} \gamma^{W} \circ \gamma^{\prime \prime}: H\left(x_{V}, x_{V}\right) \rightarrow F \lim ^{W} Q^{W}\left(x_{V}, x_{V}\right)
$$

then clearly is the desired one.

Proof of Theorem 13.4. It is readily verified that the natural equivalence

$$
\beta: R(S(x), \mathrm{Z}) \rightarrow Q(x, T(\mathrm{Z}))
$$

induces a natural equivalence

$$
\beta^{W}: R^{W}\left(S_{V}\left(X_{V}\right), \mathrm{Z}_{V}\right) \rightarrow Q^{W}\left(X_{V}, T_{V}\left(\mathrm{Z}_{V}\right)\right)
$$

given by $\beta^{W}(K, L) v^{\wedge}=\beta\left(K V_{1}, L V_{2}\right)$ for every object $K \in X_{V}$, and $L \in \mathcal{Z}_{V}$ and every map $v: V_{1} \rightarrow V_{2} \in \mathcal{V}$. Composition of $\beta^{W}$ with the functor $\lim ^{W}: \mathfrak{L}^{W} \rightarrow \mathfrak{L}$ then yields the desired natural equivalence

$$
\beta^{\prime}=\lim ^{W} \beta^{W}: \lim ^{W} R^{W}\left(S_{V}\left(X_{V}\right), \mathrm{Z}_{V}\right) \rightarrow \lim ^{W} Q^{W}\left(x_{V}, T_{V}\left(\mathrm{Z}_{V}\right)\right) .
$$

The proof of Theorem 13.5 is similar.

For the proof of Theorem 13.8 we need the following lemma.

Lemma 13.10. Let $R: \mathrm{Z}, \mathrm{Z} \rightarrow \mathfrak{L}$ be a hom-functor rel. $F$. and let $W$ be a proper category. If the category $\mathrm{Z}$ is $W$-direct and the functor $F: \mathfrak{L} \rightarrow \mathfrak{M}$ is true and $W$-inverse, then there exists a natural equivalence

$$
\phi_{R}: R\left(\lim _{W}\left(\mathrm{Z}_{W}\right), \mathrm{Z}\right) \rightarrow \lim ^{W} R^{W}\left(\mathrm{Z}_{W}, \mathrm{Z}\right) .
$$

Proof of Theorem 13.8. It is readily verified that the natural equivalence

$$
\beta: R(S(x, y), \mathcal{Z}) \rightarrow Q(x, T(Y, Z))
$$

induces a natural equivalence

$$
\beta^{W}: R^{W}\left(S_{W}\left(X^{V}, Y_{V}\right), \mathrm{Z}\right) \rightarrow Q^{W}\left(\mathfrak{X}^{V}, T^{v}\left(Y_{V}, \mathrm{Z}\right)\right)
$$

given by $\beta^{w}(K, L, Z) v^{\wedge}=\beta\left(K V_{2}, L V_{1}, Z\right)$ for every object $K \in \mathfrak{X}^{v}, L \in \mathcal{Y}_{V}$ and $Z \in Z$ and every map $v: V_{1} \rightarrow V_{2} \in \mathcal{V}$. Then composition of the functor $\lim ^{W}: \mathfrak{L}^{W} \rightarrow \mathfrak{L}$ with $\beta^{W}$ and of the functor $S_{W}\left(\mathfrak{X}^{V}, Y_{V}\right)$ with $\phi_{R}$ (see Lemma 13.10) yields natural equivalences

$$
\begin{aligned}
& \lim ^{W} \beta^{W}: \lim ^{W} R^{W}\left(S_{W}\left(\mathscr{X}^{V}, \mathcal{Y}_{V}\right), \mathrm{Z}\right) \rightarrow \lim ^{W} Q^{W}\left(\mathfrak{X}^{v}, T^{v}\left(\mathcal{Y}_{v}, \mathrm{Z}\right)\right), \\
& \left.\phi_{R} S_{W}: R\left(\lim _{W} S_{W}\left(x^{V}, y_{V}\right), \mathrm{z}\right) \rightarrow \lim ^{W} R^{W}\left(S_{W}\left(x^{V}, y_{V}\right), \mathrm{z}\right)\right)
\end{aligned}
$$

and the theorem follows by putting

$$
\beta_{0}=\lim ^{W} \beta^{W} \circ \phi_{R} S_{W} .
$$

Proof of Lemma 13.10. We first consider the case where $\mathfrak{L}=\mathfrak{T}, F$ $=E: \mathfrak{T} \rightarrow \mathfrak{T}$, the identity functor and $R=H: \mathrm{Z}, \mathrm{Z} \rightarrow \mathcal{L}$. Composition of the natural transformation

$$
\lambda_{W}: E\left(\mathrm{Z}_{W}\right) \rightarrow E_{W} \lim _{W}\left(\mathrm{Z}_{W}\right)
$$

with the functor $H^{W}: Z_{W}, \mathrm{Z} \rightarrow \mathfrak{L}^{W}$ yields a natural transformation 


$$
H^{W} \lambda_{W}: H^{W}\left(E_{W} \lim _{W}\left(\mathrm{Z}_{W}\right), \mathrm{Z}\right) \rightarrow H^{W}\left(\mathrm{Z}_{W}, \mathrm{Z}\right) .
$$

Denote by

$$
\phi_{H}: H\left(\lim _{W}\left(\mathrm{Z}_{W}\right), \mathrm{Z}\right) \rightarrow \lim ^{W} H^{W}\left(\mathrm{Z}_{W}, \mathrm{Z}\right)
$$

the unique natural transformation such that commutativity holds in the diagram

$$
\begin{gathered}
E^{W} H\left(\lim _{W}\left(\mathrm{Z}_{W}\right), \mathrm{Z}\right) \stackrel{i}{\longrightarrow} H^{W}\left(E_{W} \lim _{W}\left(\mathrm{Z}_{W}\right), \mathrm{Z}\right) \\
\downarrow E^{W} \phi_{H} \\
E^{W} \lim ^{W} H^{W}\left(\mathrm{Z}_{W}, \mathrm{z}\right) \stackrel{\lambda^{W} H^{W}}{\longrightarrow} H^{W}\left(\mathrm{Z}_{W}, \mathrm{Z}\right)
\end{gathered}
$$

where $i$ is the identity. It then may be verified by straightforward computation that $\phi_{H}$ is a natural equivalence.

Replacing everywhere $H$ by $R$ we obtain a unique natural transformation

$$
\phi_{R}: R\left(\lim _{W}\left(\mathrm{Z}_{W}\right), \mathrm{Z}\right) \rightarrow \lim ^{W} R^{W}\left(\mathrm{Z}_{W}, \mathrm{Z}\right)
$$

such that commutativity holds in the diagram obtained from (13.11) by replacing $H$ by $R$. Because $R$ is a hom-functor rel. $F$ there exists a natural equivalence

$$
\delta: H(\mathrm{Z}, \mathrm{Z}) \rightarrow F R(\mathrm{Z}, \mathrm{Z}) .
$$

This induces a natural equivalence

$$
\delta^{W}: H^{W}\left(\mathrm{Z}_{W}, \mathrm{Z}\right) \rightarrow F^{W} R^{W}\left(\mathrm{Z}_{W}, \mathrm{Z}\right)
$$

given by $\delta^{W}(K, Z) v^{\wedge}=\delta\left(K v^{\wedge}, Z\right)$ for every object $K \in Z_{W}, Z \in Z$ and $v^{\wedge} \in W$.

Now consider Figure $\mathrm{I}$, in which $i$ denotes the identity. It follows from the definitions of $\phi_{H}$ and $\phi_{R}$ that commutativity holds in the lower and upper rectangles, from the definition of $\delta^{W}$ that commutativity holds in the big rectangle and in $(B)$. Because $F$ is $W$-inverse commutativity also holds in $(A)$ and consequently

$$
\lambda^{W} H^{W} \circ E^{W} \phi_{H}=\lambda^{W} H^{W} \circ\left(E^{W} \lim ^{W} \delta^{W}\right)^{-1} \circ E^{W} \chi R^{W} \circ E^{W} F \phi_{R} \circ E^{W} \delta \lim _{W} .
$$

Hence in view of the uniqueness of $\phi_{H}$

$$
\phi_{H}=\left(\lim ^{W} \delta^{W}\right)^{-1} \circ \chi R^{W} \circ F \phi_{R} \circ \delta \lim _{W} .
$$

As $\delta, \chi$ and $\phi_{H}$ are natural equivalences it follows that $F \phi_{R}$ is so. Because $F$ is true this implies that $\phi_{R}$ is also a natural equivalence. This completes the proof.

14. The functor $H$. Let $Z$ be a category. It will be shown that a sufficient condition in order that the functor $H: \mathrm{Z}, \mathrm{Z} \rightarrow \mathfrak{M}$ has a left adjoint is that the category $Z$ has direct limits. It then follows from Theorem 12.3 that, for every proper category $v$, the lifted functor $H^{v}: Z_{V}, Z \rightarrow \mathbb{T}^{v}$ also has a left adjoint. 


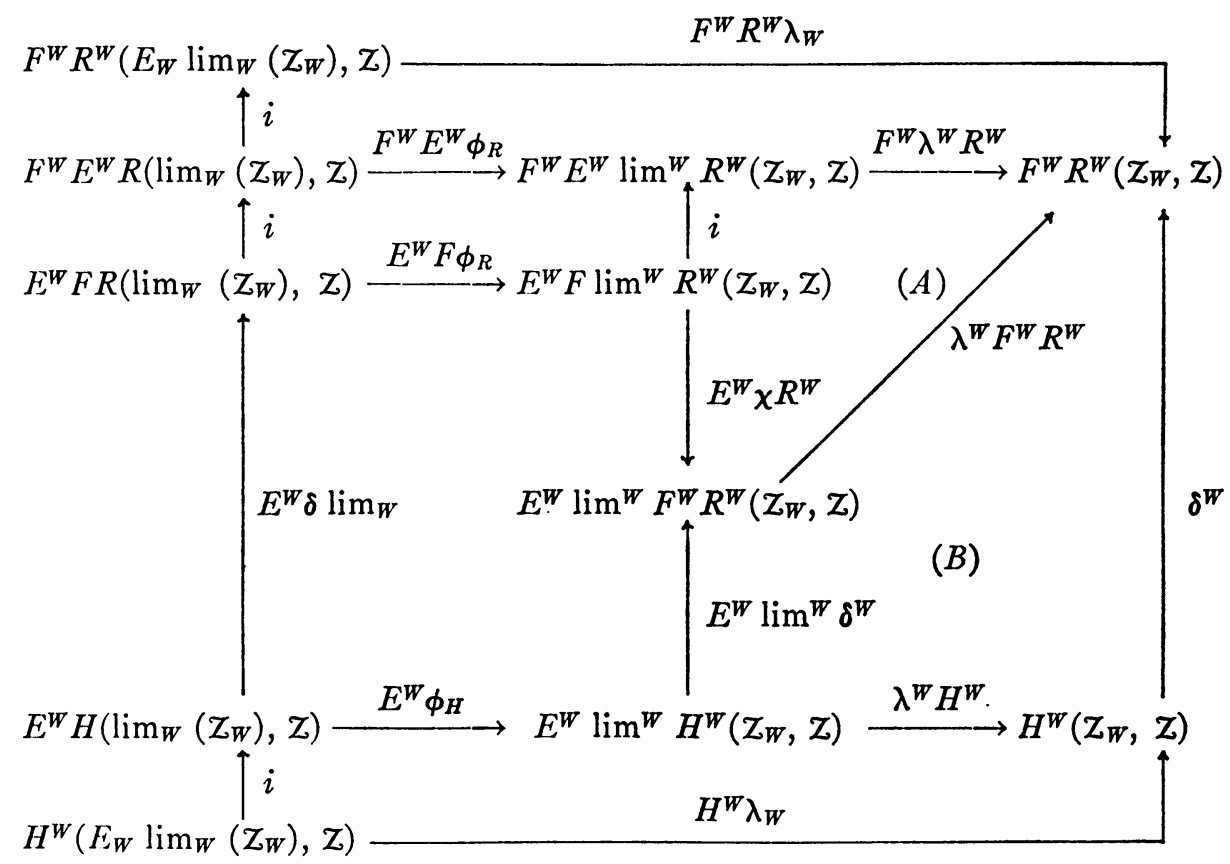

FIGURE I

The converse also holds, i.e. if for every property category $v$ the lifted functor $H^{v}: \mathrm{Z}_{V}, \mathrm{Z} \rightarrow \mathfrak{M}$ has a left adjoint, then $\mathrm{Z}$ has direct limits. Several known functors involving c.s.s. complexes may be obtained from $H^{V}\left(\boldsymbol{Z}_{V}, \mathbf{Z}\right)$ for suitable categories $\mathcal{V}$ and $Z$ or from a left adjoint of such a functor. These applications will be dealt with in [5].

Let $V$ be a proper category. With each object $C \in \mathfrak{M}^{V}$ we associate a proper category $\mathfrak{C}$, defined as follows. The objects of $\mathfrak{e}$ are the pairs $(V, c)$ where $V \in \mathcal{V}$ is an object and $c \in C V$. The maps of $\mathcal{C}$ are the triples $\left(v, c_{1}, c_{2}\right)$ where $v: V_{1} \rightarrow V_{2} \in v$ is a map, $c_{1} \in C V_{1}, c_{2} \in C V_{2}$ and $(C v) c_{1}=c_{2}$; the domain of $\left(v, c_{1}, c_{2}\right)$ is $\left(V_{1}, c_{1}\right)$ and the range is $\left(V_{2}, c_{2}\right)$. If $\left(v^{\prime}, c_{2}, c_{3}\right):\left(V_{2}, c_{2}\right) \rightarrow\left(V_{3}, c_{3}\right)$ is another map, then composition is defined by

$$
\left(v^{\prime}, c_{2}, c_{3}\right) \circ\left(v, c_{1}, c_{2}\right)=\left(v^{\prime} \circ v, c_{1}, c_{3}\right) .
$$

A map $a: C \rightarrow D \in \mathscr{T}^{V}$ induces a covariant functor $a^{0}: \mathcal{C} \rightarrow D$ defined by

$$
\begin{aligned}
a^{0}(V, c) & =(V,(a V) c), \\
a^{0}\left(v, c_{1}, c_{2}\right) & =\left(v,\left(a V_{1}\right) c_{1},\left(a V_{2}\right) c_{2}\right)
\end{aligned}
$$

for every object $(V, c)$ and map $\left(v, c_{1}, c_{2}\right):\left(V_{1}, c_{1}\right) \rightarrow\left(V_{2}, c_{2}\right)$ in $\mathbb{e}$.

For every object $C \in \mathbb{M}^{V}$ define a covariant functor $C^{0}: \mathcal{C} \rightarrow \circlearrowright$ by restriction to the first coordinate, i.e. 


$$
C^{0}(V, c)=V, \quad C^{0}\left(v, c_{1}, c_{2}\right)=v
$$

for every object $(V, c)$ and map $\left(v, c_{1}, c_{2}\right)$ in e. Clearly for each map $a: C \rightarrow D$ $\in \mathbb{M}^{v}$

$$
D^{0} a^{0}=C^{0} .
$$

Now define a covariant functor

$$
\otimes_{d}: \mathfrak{N}^{V}, \mathrm{Z}_{V} \rightarrow \mathrm{Z}_{d}
$$

as follows; for each object $C \in \mathfrak{M}^{v}$ and $K \in Z_{V}$.

$$
C \otimes_{d} K=\left(\mathfrak{e}, K C^{0}\right)
$$

where $K C^{0}: \mathrm{C} \rightarrow Z$ denotes the composite functor; for every map $a: C \rightarrow D \in \mathfrak{M}^{v}$ and $k: K \rightarrow K^{\prime} \in \mathrm{Z}_{V}$

$$
a \otimes_{d} k=\left(a^{0}, k C^{0}\right)
$$

where $k C^{0}: K C^{0} \rightarrow K^{\prime} D^{0} a^{0}$ is the natural transformation from the composite functor $K C^{0}: \mathrm{e} \rightarrow \mathrm{Z}$ to the composite functor $K^{\prime} D^{0} a^{0}=K^{\prime} C^{0}: \mathrm{e} \rightarrow \mathrm{Z}$.

THEOREM 14.1. A category $Z$ has direct limits if and only if for every proper category $v$ there exists a natural equivalence

$$
\beta: H\left(\lim _{d}\left(\mathfrak{M}^{v} \otimes_{d} \mathrm{Z}_{V}\right), \mathrm{Z}\right) \rightarrow H\left(\mathfrak{M}^{v}, H^{v}\left(\mathrm{Z}_{V}, \mathrm{Z}\right)\right)
$$

i.e.

$$
\beta: \lim _{d}\left(\mathfrak{T N}^{V} \otimes_{d} \mathrm{Z}_{V}\right) \dashv H^{V}\left(\mathrm{Z}_{V}, \mathrm{Z}\right) .
$$

Combination of Theorem 14.1 with Theorem 12.4 yields

COROLlaRY 14.2. Let $\mathrm{Z}$ have direct limits, let $v$ be a proper category and let $W=V^{\wedge}$. Then there exists a natural equivalence

$$
\sigma: \lim _{d}\left(\mathfrak{T}^{V} \otimes_{d} \mathrm{Z}_{V}\right) \rightarrow \lim _{W} S_{W}\left(\mathfrak{T}^{V}, \mathrm{Z}_{V}\right)
$$

where $S(\mathfrak{T}, \mathrm{Z})=\lim _{d}\left(\mathfrak{T}^{0} \otimes_{d} \mathrm{Z}_{0}\right)$ is a left adjoint of the functor $H: \mathrm{Z}, \mathrm{Z} \rightarrow \mathfrak{T}$.

For the proof of Theorem 14.1 we need the following lemma.

Lemma 14.3. Let $\mathrm{Z}$ be a category and let $\mathrm{U}$ be a proper category. Then there exists a natural equivalence

$$
\gamma: H\left(\mathfrak{N}^{V} \otimes_{d} \mathrm{Z}_{V}, E_{d}(\mathrm{Z})\right) \rightarrow H\left(\mathfrak{T}^{V}, H^{V}\left(\mathcal{Z}_{V}, \mathrm{Z}\right)\right) .
$$

Proof of Theorem 14.1. Let $Z$ have direct limits. Composition of the natural equivalence $\alpha_{d}$ : $\lim _{d} \dashv E_{d}$ with the functor $\otimes_{d}$ yields a natural equivalence

$$
\alpha_{d} \otimes_{d}: H\left(\lim _{d}\left(\mathfrak{T}^{V} \otimes_{d} \mathrm{Z}_{V}\right), \mathrm{Z}\right) \rightarrow H\left(\mathfrak{N}^{v} \otimes_{d} \mathrm{Z}_{V}, \mathrm{E}_{d}(\mathrm{Z})\right)
$$

Clearly the composite natural equivalence 


$$
\beta=\gamma \circ \alpha_{d} \otimes_{d}: H\left(\lim _{d}\left(\mathscr{T}^{V} \otimes_{d} \mathcal{Z}_{V}\right), \mathrm{Z}\right) \rightarrow H\left(\mathscr{T}^{V}, H^{V}\left(Z_{V}, \mathrm{Z}\right)\right)
$$

then is the desired one.

Now suppose that for every proper category $v$ a natural equivalence

$$
\beta: H\left(\lim _{t}\left(\mathfrak{M}^{V} \otimes_{d} \mathrm{Z}_{V}\right), \mathrm{Z}\right) \rightarrow H\left(\mathfrak{M}^{v}, H^{V}\left(\mathrm{Z}_{V}, \mathrm{Z}\right)\right)
$$

is given. Let $P \in \mathbb{M}$ be a set consisting of one element $p$. Let $K \in \mathcal{Z}_{V}$ and $Z \in \mathcal{Z}$ be objects. An element $f \in H\left(E^{v} P, H^{v}(K, Z)\right)$ then is a function which assigns to every object $V \in V$ a map $f V: P \rightarrow H(K V, Z)$ subject to certain naturality conditions. Denote by $\delta f \in H\left(K, E_{V} Z\right)$ the map defined by $(\delta f) V=(f V) p$ for every object $V \in \mathcal{V}$. It then is readily verified that the function

$$
\delta: H\left(E^{v} P, H^{v}\left(\mathrm{Z}_{V}, \mathrm{Z}\right)\right) \rightarrow H\left(\mathrm{Z}_{V}, E_{V} \mathrm{Z}\right)
$$

so defined is a natural equivalence. Now composition of the natural equivalence $\beta$ with $\delta$ yields a natural equivalence

$$
\delta \circ \beta\left(E^{V} P\right): H\left(\lim _{d}\left(E^{V} P \otimes_{d} \mathrm{Z}_{V}\right), \mathrm{Z}\right) \rightarrow H\left(\mathrm{Z}_{V}, E_{V} \mathrm{Z}\right) .
$$

Hence $\mathrm{Z}$ is $\boldsymbol{V}$-direct. This completes the proof.

Proof of Lemma 14.3. Let $C \in \mathfrak{N}^{v}, K \in \mathrm{Z}_{V}$ and $Z \in \mathrm{Z}$ be objects. For every map

$$
\left(O_{C}, f\right): C \otimes_{d} K=\left(\mathfrak{e}, K C^{0}\right) \rightarrow E_{d} Z=\left(\mathcal{\Theta}, E_{0} Z\right)
$$

in $Z_{d}$ define a map $\gamma\left(O_{C}, f\right): C \rightarrow H^{v}(K, Z)$ in $\mathfrak{T}^{v}$ by

$$
\left(\gamma\left(O_{c}, f\right) V\right) c=f(V, c)
$$

for every object $(V, c) \in \mathcal{e}$. It then may be verified by straightforward computation that the function $\gamma\left(O_{C}, f\right)$ so defined is an equivalence in $\mathfrak{T}^{\boldsymbol{V}}$ and that the function

$$
\gamma: H\left(\mathscr{T}^{v} \otimes_{d} \mathrm{Z}_{V}, E_{d}(\mathrm{Z})\right) \rightarrow H\left(\mathscr{T}^{v}, H^{V}\left(\mathrm{Z}_{V}, \mathrm{Z}\right)\right)
$$

so defined is a natural equivalence.

\section{BIBLIOGRAPHY}

1. H. Cartan and S. Eilenberg, Homological algebra, Princeton University Press, 1956.

2. S. Eilenberg and S. MacLane, General theory of natural equivalences, Trans. Amer. Math. Soc. vol. 58 (1945) pp. 231-294.

3. S. Eilenberg and N. Steenrod, Foundations of algebraic topology, Princeton University Press, 1952.

4. I. M. James, On the suspension triad, Ann. of Math. vol. 63 (1956) pp. 191-247.

5. D. M. Kan, Functors involving c.s.s. complexes, Trans. Amer. Math. Soc. vol. 87 (1958) pp. $330-346$. 505.

6. J.-P. Serre, Homologie singulière des espaces fibres, Ann. of Math. vol. 54 (1951) pp. 425-

Columbia University, NEW YORK, N. Y. 